\title{
MeV-scale sterile neutrino decays at the Fermilab Short-Baseline Neutrino program
}

\author{
Peter Ballett, Silvia Pascoli and Mark Ross-Lonergan \\ Institute for Particle Physics Phenomenology, Department of Physics, Durham University, \\ South Road, Durham DH1 3LE, United Kingdom \\ E-mail: peter.ballett@durham.ac.uk, silvia.pascoli@durham.ac.uk, \\ mark.ross-lonergan@durham.ac.uk
}

ABSTRACT: Nearly-sterile neutrinos with masses in the MeV range and below would be produced in the beam of the Short-Baseline Neutrino (SBN) program at Fermilab. In this article, we study the potential for SBN to discover these particles through their subsequent decays in its detectors. We discuss the decays which will be visible at SBN in a minimal and non-minimal extension of the Standard Model, and perform simulations to compute the parameter space constraints which could be placed in the absence of a signal. We demonstrate that the SBN programme can extend existing bounds on well constrained channels such as $N \rightarrow \nu l^{+} l^{-}$and $N \rightarrow l^{ \pm} \pi^{\mp}$ while, thanks to the strong particle identification capabilities of liquid-Argon technology, also place bounds on often neglected channels such as $N \rightarrow \nu \gamma$ and $N \rightarrow \nu \pi^{0}$. Furthermore, we consider the phenomenological impact of improved event timing information at the three detectors. As well as considering its role in background reduction, we note that if the light-detection systems in SBND and ICARUS can achieve nanosecond timing resolution, the effect of finite sterile neutrino mass could be directly observable, providing a smoking-gun signature for this class of models. We stress throughout that the search for heavy nearly-sterile neutrinos is a complementary new physics analysis to the search for eV-scale oscillations, and would extend the BSM programme of SBN while requiring no beam or detector modifications.

Keywords: Beyond Standard Model, Neutrino Physics

ARXIV EPRINT: 1610.08512 


\section{Contents}

1 Introduction 1

2 Sterile neutrino production and decay 4

2.1 Production at BNB 5

2.2 Decay at SBN 6

2.2.1 Minimal model 6

2.2.2 Non-minimal models $\quad 9$

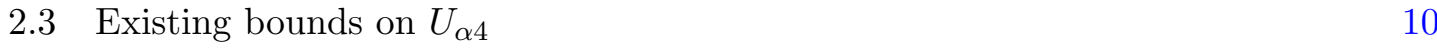

3 Simulation of SBN 13

$\begin{array}{ll}3.1 \text { Background reduction } & 15\end{array}$

$\begin{array}{ll}3.2 \text { Role of event timing } & 16\end{array}$

$\begin{array}{lll}3.3 & \text { Event spectra } & 19\end{array}$

4 Results $\quad 19$

4.1 Limits on sterile neutrino mixing 21

4.2 Timing information to study an observed signal 24

$\begin{array}{llr}5 & \text { Conclusions } & 28\end{array}$

$\begin{array}{ll}\text { A Decay rates in the minimal model } & 30\end{array}$

$\begin{array}{ll}\text { B Potential backgrounds } & 30\end{array}$

$\begin{array}{ll}\text { C PS-191 bound reproduction } & 33\end{array}$

\section{Introduction}

The neutrino sector of the Standard Model (SM) is known to be incomplete. The observation of oscillatory behaviour between neutrino flavour states [1] suggests that neutrinos possess a mass matrix with off-diagonal terms in the flavour basis. There are many models that have been invoked in the literature to explain this observation as well as the lightness of neutrino masses, ranging from the ever popular see-saw mechanisms [2-4] to radiative mass generation $[5,6]$ or even more involved constructions such as neutrino masses originating from extra-dimensions [7]. It will ultimately be the role of phenomenology to find ways to distinguish between potential candidate models, and explore what can be deduced about the completion of the neutrino sector from the analysis of contemporary experiments. A common, although not necessary, feature in Beyond the SM (BSM) models which account for neutrino masses is the presence of sterile neutrinos, SM-gauge singlet 
fermions which couple to the active neutrinos via Yukawa interactions. ${ }^{1}$ After electroweak symmetry breaking, these particles are coupled bilinearly to the active neutrino fields, and in the mass basis, we find an extended neutrino sector including new states with mixingsuppressed gauge interactions. A priori their mass and interaction scales can span many orders of magnitude, leading to a wide range of distinct observable phenomena. One of the best known examples is the short-baseline oscillation signature associated with a sterile neutrino mass around the eV-scale (see e.g. ref. [8] for a recent review), which has been invoked to explain anomalies found at some short-baseline oscillation experiments [9-11]. Explaining all data in an economical fashion appears challenging in these models [12, 13], but more results would be needed before a decision can be made as to their role in the neutrino sector. The Fermilab SBN [14] program was primarily designed to perform such a conclusive test.

The SBN experiment is comprised of three detectors placed in the Booster Neutrino Beam (BNB) at different (short) baseline distances: SBND (previously known as LAr1$\mathrm{ND}$ ) at $110 \mathrm{~m}$ from the target, MicroBooNE at $470 \mathrm{~m}$ and ICARUS at $600 \mathrm{~m}$. All three detectors employ Liquid Argon Time Projection Chamber (LArTPC) technology [15] with strong event reconstruction capabilities allowing for a significantly improved understanding of background processes compared to predecessor technologies. With this design, SBN has been shown to be able to extend the current bounds on light oscillating sterile neutrinos, thoroughly exploring the $\mathrm{eV}$-scale sterile neutrino mass region, whilst also pursuing many other physics goals [14].

In this article, we assess SBN's potential to contribute to the search for sterile neutrinos, in a manner complementary to the oscillatory analysis. The new fermions in our study are assumed to have masses around the $\mathrm{MeV}$ scale. These particles are light enough to be produced in neutrino beams via meson decay, but have masses sufficiently large to prevent oscillatory effects with the active neutrinos through loss of coherence (see e.g. ref. [16]), instead propagating long distances along the beamline. Due to the presence of mixing they are unstable, and their subsequent decay products can be observed in neutrino detectors. We stress that the search for $\mathrm{MeV}$-scale sterile neutrinos is entirely compatible with the primary goals of SBN, and requires modification of neither the beam nor detector designs.

The reconstruction $[17,18]$, energy resolution [19] and excellent calorimetric particle identification capabilities of LAr [20] technology means the SBN program provides an ideal scenario to study this "decay-in-flight" of sterile neutrinos. This technology allows for a high degree of background suppression on well studied decay modes while also allowing the study of channels which have been poorly bounded by similar experiments due to large backgrounds and challenging signals. For example, the differentiation between an electron- or photon- induced EM shower can be achieved by studying their rate of energy loss in the first $3 \mathrm{~cm}$ of their ionising track [21]. Furthermore, as we discuss in section 3.2, if a sufficiently good timing resolution of scintillation light is achieved, the timing structure of markedly sub-luminal sterile neutrinos can be utilised as both a rejection mechanism for beam related backgrounds as well as a further aid for model discrimination and mass measurement.

\footnotetext{
${ }^{1}$ We focus on mass eigenstates which are nearly sterile but mix with small angles with the active ones. For simplicity, and following previous literature, we call them "sterile neutrinos" throughout the text.
} 


\begin{tabular}{|l|l|l|l|l|}
\hline & PS-191 & SBND & MicroBooNE & ICARUS \\
\hline POT & $0.86 \times 10^{19}$ & $6.6 \times 10^{20}$ & $13.2 \times 10^{20}$ & $6.6 \times 10^{20}$ \\
\hline Volume & $216 \mathrm{~m}^{3}$ & $80 \mathrm{~m}^{3}$ & $62 \mathrm{~m}^{3}$ & $340 \mathrm{~m}^{3}$ \\
\hline Baseline $^{-2}$ & $(128 \mathrm{~m})^{-2}$ & $(110 \mathrm{~m})^{-2}$ & $(470 \mathrm{~m})^{-2}$ & $(600 \mathrm{~m})^{-2}$ \\
\hline Ratio/PS-191 & - & 38.5 & 3.3 & 5.5 \\
\hline S/ $\sqrt{B}$ Ratio & - & 16.3 & 1.8 & 1.1 \\
\hline
\end{tabular}

Table 1. A comparison of the relative exposure at each SBN detector compared to PS-191. One would expect all three SBN detectors to see increased numbers of events than PS-191 did, with SBND seeing the largest enhancement of a factor of 38.5. The final row takes into account the scaling in masses leading to increased backgrounds, although the achievable reconstruction of LAr should reduce these significantly.

We restrict our analysis to sterile neutrino masses below the kaon mass. Kaons and pions are produced in large numbers at $\mathrm{BNB}$, and their subsequent decays will generate a flux of sterile neutrinos. In this mass range, the strongest bounds on sterile neutrinos which mix with electron and muon neutrinos come from PS-191 [22, 23], a beam dump experiment which ran at CERN in 1984. PS-191 was constructed from a helium filled flash chamber decay region, followed by interleaved iron plates and EM calorimeters. It was located $128 \mathrm{~m}$ downstream of a beryllium target and $2.3^{\circ}$ (40 mrads) off-axis, obtained $0.86 \times 10^{19}$ POT over the course of its run-time, and had a total detector volume of $6 \times 3 \times 12=216 \mathrm{~m}^{3}$. We can estimate the sensitivity of the three SBN detectors and how they will compare to PS-191 by estimating the experiments' exposure, defined here as POT $\times \mathrm{Vol} \times R^{-2}$. We compare the three detectors to PS-191 in table 1, which indicates that all detectors of the SBN complex expect a larger exposure, with SBND seeing the greatest enhancement by a factor of around 40. In addition to the larger exposure, there is also an enhancement of the expected decay events at SBN due to its lower beam energy. The sterile neutrinos at SBN are produced by the $8 \mathrm{GeV}$ BNB beam and have a softer spectrum than those produced by the $19.2 \mathrm{GeV}$ CERN Proton Synchrotron beam used at PS-191. As we discuss in more detail in section 2 , the probability that the sterile neutrino decays inversely scales with momentum, $1 /\left|P_{N}\right|$, and we would therefore expect any BNB detectors to see more events than PS-191 for equivalent neutrino exposures.

However, exposure alone does not dictate sensitivity. PS-191 was purposefully built to search for decays of heavy fermions. To minimise the background induced by active neutrino scattering, the total mass of the detector (and therefore number of target nuclei) was chosen to be small (approximately 20 ton). Conversely, the SBN detectors were designed to search for neutrino interactions and thus have significantly larger masses (112, 66.6 and 476 tons respectively). SBN will not only see a greater number of decay events than PS-191 but also a greater background for a given exposure. Therefore, the degree of background reduction will be crucial in determining its ultimate performance. We return to this issue in section 3.1.

The paper is structured as follows. In section 2 we present an overview of sterile neutrino decay in minimal and non-minimal models relevant for beam dump experiments. 
We then present the details of our simulation in section 3 and show illustrative event spectra for some channels of interest. In section 4, we present and discuss the exclusion contours that SBN could place on the model in the absence of a signal. We then study how the event timing information could be used to test the hypothesis of sterile neutrino decay-in-flight and to help constrain the particle masses if a positive signal were detected. We make some concluding remarks in section 5 .

\section{Sterile neutrino production and decay}

The most general renormalizable lagrangian extending the SM to include a new gaugesinglet fermion $N$ is given by

$$
\mathcal{L}_{N}=\mathcal{L}_{\mathrm{SM}}+\bar{N} i \not N+\left(\frac{m_{N}}{2} \overline{N^{\mathrm{c}}} N+y_{\alpha} \bar{L}_{\alpha} H N+\text { h.c. }\right),
$$

where $N^{c}=C \bar{N}^{\mathrm{T}}$ with $C$ denoting the charge-conjugation matrix, $L_{\alpha}$ is the SM leptonic doublet of flavour $\alpha, y_{\alpha}$ represents the Yukawa couplings and $m_{N}$ a Majorana mass term for $N$. The extension to multiple new fermions involves promoting $y$ and $m_{N}$ to matrices with indices for the new states, but will offer no real phenomenological difference in the following analysis. $^{2}$ Much work has been done understanding the phenomenology of such novel neutral states, which varies significantly over their large parameter spaces. Lagrangians similar to this have been used in the literature for a wide range of purposes. If the new particle has a mass around $10^{12}-10^{15} \mathrm{GeV}$ it could provide a natural way to suppress the size of active neutrino masses through the Type I or III see-saw mechanisms [2-4]. A lighter neutral fermion, with a mass around the $\mathrm{keV}$ scale, remains a promising dark matter candidate [24]. A synthesis of these ideas is found in the so-called $\nu \mathrm{MSM}$ which simultaneously can explain dark matter, neutrino masses and successful baryogenesis [25]. If we consider sterile neutrinos at even lower energy scales, with masses at the eV scale or below, these particles can alter the neutrino oscillation probability, leaving observable signatures at oscillation experiments. Indeed, such particles have been proposed to alleviate short-baseline oscillation anomalies; although, no minimal solution seems to provide a compelling universal improvement to the current data $[12,13]$.

A key feature of models of sterile neutrinos are the weaker-than-weak interactions which arise from mass mixing. In the minimal lagrangian in eq. (2.1), the only direct couplings to new sterile flavour eigenstates are neutrino-Higgs interactions. However, these couplings generate off-diagonal neutrino bilinears below the electroweak symmetry breaking scale, leading to mixing-mediated interactions with SM gauge bosons for the mostly neutral mass eigenstate. This allows them to be produced in and decay via SM gauge interactions, albeit suppressed by the mixing angle.

The possibility remains that extra particles exist beyond the minimal lagrangian and these mediate other interactions, either directly with SM fields or, as before, via mixing.

\footnotetext{
${ }^{2}$ The minimal single $N$ extension does not allow for the observed masses of the neutrinos, as the mass matrix is rank 1. We assume that an appropriate extension has been introduced to satisfy neutrino oscillation data while introducing no new dynamics at the lower energy scales of interest.
} 
Throughout our work, we assume that the production of $N$, described in section 2.1, is generated by the interactions in eq. (2.1). However, we will return to the idea of a nonminimal lagrangian in section 2.2.2 when discussing the decay modes of $N$.

\subsection{Production at BNB}

For sterile neutrinos which are light enough to be produced from a meson beam, there is a qualitative divide in the phenomenology somewhere between $\mathrm{keV}$ and $\mathrm{eV}$ masses. ${ }^{3}$ If the sterile neutrinos are massive enough for their mass-splittings with the light neutrinos to be larger than the wavepacket energy-uncertainty associated with the production mechanism, they no longer oscillate [16]. Neutral particles produced in the beam will propagate towards the detector and may be observed by their subsequent decay into SM particles. Experiments seeking to measure such decays are generally known as beam dump experiments, where proton collisions with a target produce particles to be observed down-wind of the source [22, 23, 26-30]. It has been pointed out that the difference between a beam dump and a conventional neutrino beam is more a matter of philosophy than design, and we can expect many experiments to have some sensitivity to novel heavy states [31-33]. For the BNB, we can estimate the mass at which the oscillatory behaviour is suppressed as follows: the decay pipe for BNB is around $50 \mathrm{~m}$ in length, which is considerably shorter than the decay lengths of the mesons in the beam, and we assume that this length defines the wavepacket width at production. The relevant parameter is the decoherence parameter $[16,34] \xi=2 \pi \frac{\lambda_{\mathrm{d}}}{\lambda_{\nu}}$, where $\lambda_{\mathrm{d}}=50 \mathrm{~m}$ and $\lambda_{\nu}$ is the standard neutrino oscillation length $\lambda_{\nu}=\Delta m^{2} / 4 E_{\nu}$. For $\xi \gg 1$ the wave packet is insufficiently broad to accommodate a coherent superposition of the heavy and light neutrino states. We estimate that this occurs for the BNB at $\Delta m^{2} \gtrsim 100 \mathrm{eV}^{2}$.

In a conventional neutrino beam, most neutrinos are derived from meson decay, and we assume in this work that the sterile neutrinos are produced from the decays of pions and kaons, restricting our sterile neutrino mass to $m_{N} \leqslant m_{K}$. Larger sterile neutrino masses could be probed by working at higher energies in the initial proton beam, where the neutral fermions could come from decays of charmed mesons such as $D^{ \pm}$. This strategy will be used by the upcoming SHiP experiment $[29,30]$ but will not be considered further in the present work as $D$ mesons are produced in extremely small numbers due to the relatively low energy of the BNB beam [35]. As such we restrict ourselves to the naturally defined mass range of interest for SBN, eV « $m_{N} \lesssim 494 \mathrm{MeV}$. We focus on $m_{N} \gtrsim \mathrm{MeV}$ scale states where the prospects for detection are greatest due to enhanced decay rates.

Although novel dynamics may lead to enhanced production rates of sterile neutrinos by alternative unconventional means, we neglect this possibility and assume that the sterile component of the BNB flux arises solely from meson (or secondary $\mu^{ \pm}$) decays. This process requires only mass-mixing from the $N-\nu$ Yukawa term in eq. (2.1). It follows that the amplitudes for these decays are related to those of the standard leptonic decays of mesons via an insertion of the mixing matrix element $U_{\alpha 4}$, and to leading order in the mass of the sterile neutrino over the meson mass, the $N$-fluxes will be a rescaling of the fluxes for the active neutrinos. However in order to account for flavour-specific effects, it is

\footnotetext{
${ }^{3}$ The precise mass range depends on details of the process under consideration.
} 
necessary to go beyond this approximation and consider the kinematic differences of heavy sterile neutrino production. The flux of sterile neutrinos produced from the decay of a given meson $M$ is approximated by

$$
\phi_{N}\left(E_{N}\right) \approx \phi_{\nu_{\alpha}}\left(E_{\nu_{\alpha}}\right)\left|U_{\alpha 4}\right|^{2} \frac{\rho\left(\delta_{M}^{a}, \delta_{M}^{i}\right)}{\delta_{M}^{a}\left(1-\delta_{M}^{a}\right)^{2}},
$$

where $\rho(a, b)=\mathcal{F}_{M}(a, b) \lambda^{\frac{1}{2}}(1, a, b)$ is a kinematic factor consisting of a term proportional to the two body phase space factor, $\lambda(x, y, z)=x^{2}+y^{2}+z^{2}-2(x y+y z+x z)$ and a term proportional to the matrix element, $\mathcal{F}_{M}(a, b)=a+b-(a-b)^{2}$, with $\delta_{M}^{a(i)}=m_{l_{a}\left(\nu_{i}\right)}^{2} / M^{2}[36]$.

The kinematic factor leads to two effects. First, it provides a threshold effect of suppressing the production when the phase space decreases near a kinematic boundary. Secondly, it allows for the helicity un-suppression of channels which in a conventional beam are highly suppressed. For example, the decay $\pi^{ \pm} \rightarrow e^{ \pm} \nu_{e}$ which is significantly suppressed compared to the muonic channel, sees no such suppression when the neutrino is replaced with $N$. This kinematic effect for the pion and kaon can be substantial, for $\pi \rightarrow e \nu$ this factor can be as large as $10^{5}$, which more than compensates for the significantly smaller intrinsic flux of $\nu_{e}$ intrinsic to the BNB, which is around $0.52 \%$ of the total flux [35]. The approximation in eq. (2.2) starts to fail as the mass of the sterile neutrino increases, and we begin to see components of the active flux having energies less than the sterile mass which are truncated by the kinematic factor. In order to keep the normalisation of total neutrino events constant, before $U_{\alpha 4}$ and kinematic scaling, any events which are below the sterile neutrino mass threshold are removed and the remaining flux is scaled accordingly.

\subsection{Decay at SBN}

The fermions $N$ will generally be unstable, albeit possibly long-lived, allowing for decaysin-flight into SM particles. In this work, we try to keep an open mind about the interactions of the sterile neutrino and consider all kinematically possible tree-level decays to visible SM particles for sterile fermions produced by pion and kaon decays, $10 \mathrm{MeV} \lesssim m_{N} \lesssim m_{K}$. The precise decay rates and branching ratios for these channels are model dependent. In this section, we discuss the decay rates for a minimal extension of the SM, as well as the implications of a non-minimal model.

\subsubsection{Minimal model}

We define the minimal sterile neutrino model by the Lagrangian in eq. (2.1). This encompasses the best known model of sterile neutrino phenomenology - the UV-complete Type I see-saw (and its low-scale variants) - but also provides an effective description of more complicated extensions of the SM in which the additional field content does not directly affect the neutrino sector at low energies. Decays of sterile neutrinos in such a model proceed via SM interactions suppressed by the mixing angle and have been studied in refs. [36-38]. We have plotted the branching ratios for sterile neutrinos in our mass range in figure 1, and we will now briefly summarise the decay rates most important for the present study. 

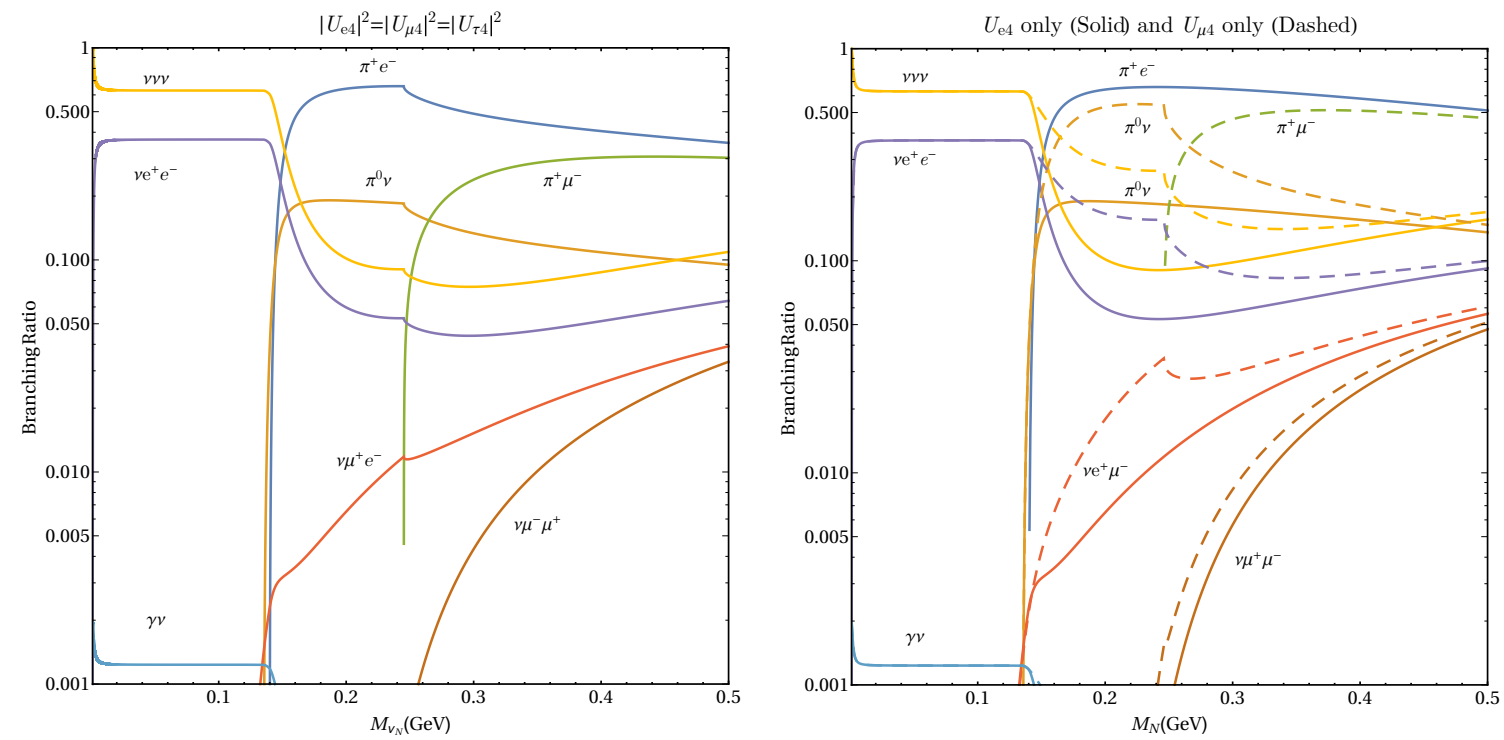

Figure 1. The branching ratios for sterile neutrino decays in the minimal 3 sterile neutrino SM extension, with masses between $1 \mathrm{MeV}$ and $0.5 \mathrm{GeV}$. We assume both a flavour independent mixing pattern (left panel) and a hierarchical scenario (right panel) in which either $U_{e 4}$ (solid lines) or $U_{\mu 4}$ (dashed lines) is the dominant mixing-matrix element.

The decays of the minimal model depend only on the mass of the $N$ and the size of neutrino mixing to various flavours, parameterized by the elements of an extended PMNS matrix, e.g. for one additional particle $U_{\alpha 4}$ for $\alpha \in\{e, \mu, \tau\}$. The branching ratios for these decays are shown in figure 1 as a function of mass for two sets of assumptions about the PMNS matrix. On the left, we show the branching ratios if all mixing elements are of a similar size, whereas on the right we assume that only $U_{\mu 4}$ or $U_{e 4}$ are non-zero. This leads to certain semi-leptonic decays being forbidden, significantly changing the phenomenology of the model for some masses.

For sterile neutrino masses less than the pion mass, the dominant decay is into three light neutrinos. This channel is for all practical purposes unobservable and we will not consider it further. The dominant decay into visible particles will be into an electronpositron pair with a branching fraction of around $38 \%$. This is true regardless of the flavour structure of the mixing matrix; although, this decay channel is not flavour-blind. If the sterile neutrino mixes primarily through $U_{e 4}$, the decay proceeds via both neutral and charged currents, but for $U_{e 4}=0$, this channel occurs via neutral current only. The decay rate for this channel is given by

$$
\begin{aligned}
\Gamma\left(N \rightarrow \nu_{\alpha} e^{+} e^{-}\right)= & \frac{G_{\mathrm{F}}^{2} m_{N}^{5}}{96 \pi^{3}}\left|U_{\alpha 4}\right|^{2}\left[\left(g_{L} g_{R}+\delta_{\alpha e} g_{R}\right) I_{1}\left(0, \frac{m_{e}}{m_{N}}, \frac{m_{e}}{m_{N}}\right)\right. \\
& \left.+\left(g_{L}^{2}+g_{R}^{2}+\delta_{\alpha e}\left(1+2 g_{L}\right)\right) I_{2}\left(0, \frac{m_{e}}{m_{N}}, \frac{m_{e}}{m_{N}}\right)\right],
\end{aligned}
$$

where $I_{1}(x, y, z)$ and $I_{2}(x, y, z)$ are integrals over phase space such that $I_{1}(0,0,0)=1$ and $I_{2}(0,0,0)=0$. Further details of the decay rates used in this work are given in appendix A. 
Although the electron-positron channel dominates the visible decays at $m_{N} \leqslant m_{\pi}^{0}$, we also consider the radiative decay $N \rightarrow \nu_{i} \gamma$ which would generate an observationally challenging single photon signal [39]. In the minimal model the decay occurs via a charged-lepton $/ W$ loop and has a rate given by

$$
\Gamma\left(N \rightarrow \nu_{i} \gamma\right)=\frac{G_{\mathrm{F}}^{2} m_{N}^{5}\left|U_{\alpha 4}\right|^{2}}{192 \pi^{3}}\left(\frac{27 \alpha}{32 \pi}\right) .
$$

This decay channel is significantly suppressed by the light mass of the sterile neutrino, the mixing-matrix elements and the loop factor. It can be estimated at around $\Gamma(N \rightarrow$ $\left.\nu_{i} \gamma\right) /(\mathrm{GeV}) \approx 10^{-20}\left(m_{N} / \mathrm{GeV}\right)^{5}$. We see in figure 1 that this leads to a branching ratio of around $10^{-3}$.

Additional leptonic decays open up for sterile neutrino masses which satisfy $m_{N} \geqslant$ $m_{\mu}+m_{e}$. Although with a smaller branching ratio, decays involving muons are clean signatures at LAr detectors. In the case of $N \rightarrow \nu_{\alpha} \mu^{+} \mu^{-}$the decay occurs by both neutral and charged currents and follows from the $N \rightarrow \nu_{\alpha} e^{+} e^{-}$decay given above with the replacement $m_{e} \rightarrow m_{\mu}$. The mixed-flavour decays, e.g. $N \rightarrow \nu_{\alpha} \mu^{ \pm} e^{ \pm}$, occur by charged current only and are given by

$$
\Gamma\left(N \rightarrow \nu_{\alpha} \beta^{-} \alpha^{+}\right)=\frac{G_{\mathrm{F}}^{2} m_{N}^{5}\left|U_{\beta 4}\right|^{2}}{192 \pi^{3}} I_{1}\left(\frac{m_{\beta}}{m_{N}}, \frac{m_{\alpha}}{m_{N}}, \frac{m_{\alpha}}{m_{N}}\right),
$$

with $\{\alpha, \beta\}=\{e, \mu\}$. The next thresholds lie just above the pion mass, where two further decays become possible: $N \rightarrow \nu \pi^{0}$ and $N \rightarrow e^{ \pm} \pi^{\mp}$. These processes quickly become the dominant decays at this mass range. The decay rate for the first process is given by

$$
\Gamma\left(N \rightarrow \nu_{i} \pi^{0}\right)=\sum_{\alpha} \frac{G_{\mathrm{F}}^{2} f_{\pi}^{2} m_{N}^{3}\left|U_{\alpha 4}\right|^{2}}{64 \pi}\left[1-\left(\frac{m_{\pi}}{m_{N}}\right)^{2}\right] .
$$

The decay into a charged pion and a lepton is an important channel, and one of the most constrained in direct decay experiments due to its clean two-body signal. Its decay rate has a similar algebraic form to the rate of $N \rightarrow \nu \pi^{0}$ with the addition of a CKM matrix element arising from the $W$-vertex,

$$
\Gamma\left(N \rightarrow l^{ \pm} \pi^{\mp}\right)=\left|U_{l 4}\right|^{2} \frac{G_{\mathrm{F}}^{2} f_{\pi}^{2}\left|V_{u d}\right|^{2} m_{N}^{3}}{16 \pi} I\left(\frac{m_{l}^{2}}{m_{N}^{2}}, \frac{m_{\pi}^{2}}{m_{N}^{2}}\right),
$$

where $I(x, y)$ is a kinematic function which away from the production threshold provides a small suppression factor of around 0.5. Further details are given in appendix A. If it is allowed by the flavour structure, the $N \rightarrow e^{ \pm} \pi^{\mp}$ channel dominates the branching ratios for sterile neutrino masses which satisfy $m_{\pi^{ \pm}} \lesssim m_{N}$. However, as it is mediated by a $W$ boson, in the absence of $U_{e 4}$ mixing, this decay would be forbidden and the decay into a neutral $\pi^{0}$ and a light neutrino would be dominant. Once the mass of the sterile fermion is above $m_{N} \gtrsim 235 \mathrm{MeV}$, the $\mu^{ \pm} \pi^{\mp}$ charged-lepton pion decay opens up. This is another strongly constrained channel, and its decay rate is already given in eq. (2.3) with $m_{l}=m_{\mu}$. Although this decay rate can also be arbitrarily suppressed by reducing the size of $U_{\mu 4}$, due to the constraint that all sterile neutrinos must be produced through $U_{\mu 4}$ or $U_{e 4}$ mixing, in no case will both of the $l^{ \pm} \pi^{\mp}$ channels be absent. As can be seen in the right panel of figure 1 , we can expect one of them to dominate for higher masses. 


\subsubsection{Non-minimal models}

In the previous section we discussed the decay rates for the minimal model of eq. (2.1). Although such low-scale see-saw models provide a viable and phenomenologically interesting region of parameter space for both masses and mixing, they lack a theoretically appealing mechanism to explain the sub-electroweak sterile neutrino mass scale and the sizes of active neutrino masses. Alternative models exist which feature light neutral particles, but these rely on additional fields or symmetries to help explain these scales. Indeed it has been stressed before [40] that the discovery of a light sterile neutrino would necessitate not just the addition of new neutral fermions to the SM but would be a sign of the existence of some non-trivial new physics with which to stabilise the mass scale.

If heavy novel fields are present in the full model, we can view eq. (2.1) as the renormalizable terms of an effective lagrangian. The effective field theory of a SM extension involving new sterile fermions has been considered at dimension 5 [40, 41], dimension 6 [40] and dimension 7 [42]. We extend the field content of the SM by a set of sterile fermions $N_{i}$. The lagrangian can then be decomposed as a formal power series of terms of increasing dimension $d$, suppressed by a new physics energy scale $\Lambda$,

$$
\mathcal{L}=\mathcal{L}_{N}+\sum_{d=5}^{\infty} \frac{1}{\Lambda^{d-4}} \mathcal{L}_{d}
$$

where $\mathcal{L}_{N}$ is given by eq. (2.1) as the sum of the SM and renormalizable terms including $N_{i}$. In ref. [41] the phenomenology of the effective operators at dimension 5 are considered in detail. Along with the Weinberg operator, which could be the source of a light neutrino Majorana mass term [43], the authors find two effective operators: an operator coupling the sterile neutrino to the Higgs doublet and a tensorial coupling between the sterile neutrino and the hypercharge field strength

$$
\mathcal{L}_{5} \supset \frac{a_{i j}}{\Lambda} \overline{N_{i}^{c}} N_{j}\left(H^{\dagger} H\right)+\frac{b_{i j}}{\Lambda} \overline{N_{i}{ }^{c}} \sigma^{\mu \nu} N_{j} B_{\mu \nu}
$$

At energies below the electroweak scale, and after diagonalisation into mass eigenstates for the neutrinos, these operators generate novel couplings, for example a vertex allowing $N \rightarrow h \nu\left(N_{1} \rightarrow h N_{2}\right), N \rightarrow \nu Z\left(N_{1} \rightarrow Z N_{2}\right)$ and $N \rightarrow \nu \gamma\left(N_{1} \rightarrow N_{2} \gamma\right)$ at a rate governed by the scale of new physics suppressing these operators. Of particular interest is the electroweak tensorial operator, which induces a rich range of phenomena [41]. In the mass range of interest in the present work, bounds on such an operator are fairly weak: strong constraints from anomalous cooling mechanisms in astrophysical settings apply only for lower sterile neutrino masses, whilst collider bounds only become competitive for higher masses. This could also be related to the enhanced $N \rightarrow \nu \gamma$ decay rate introduced in refs. [44, 45] to explain the short-baseline anomalous excesses. See also ref. [46] for a discussion of decay rates in the effective sterile neutrino extension up to dimension 6 .

If light degrees of freedom are present in addition to (or instead of) heavy ones, the predictions could be very different from those derived from the minimal model or the lowenergy effective theory. For example, models with sterile neutrinos that also feature novel 
interactions can have significantly different decay rates and branching fractions, strengthening some bounds and invalidating others [47]. As an example, a model with a leptophilic $Z^{\prime}$ [48] could enhance the magnitudes of some leptonic decay rates, such as $N \rightarrow \nu e^{+} \mu^{-}$, while leaving unchanged semileptonic processes like $N \rightarrow e^{ \pm} \pi^{\mp}$. Often, the bounds on masses and mixing angles in these models need to be reconsidered.

For the reasons discussed so far, it is desirable to place bounds on all possible decays of a neutral fermion allowing for non-standard decay rates to visible particles. The main consequence of this is that there is a priori no known relationship between the magnitude of the different decay rates - a single channel may be enhanced beyond its value from section 2.2.1 - and bounds inferred from the non-observation of a given channel may not hold in a non-minimal model when applied to another channel. We therefore do not restrict our study to those decays which lead to the most stringent bounds on the parameters of the minimal model, instead studying all kinematically viable decays independently.

\subsection{Existing bounds on $U_{\alpha 4}$}

The minimal lagrangian in eq. (2.1) has been the basis of many prior experimental searches for heavy sterile fermions, leading to a variety of bounds on the magnitude of the activesterile mixing relevant for sterile neutrino masses around the $\mathrm{MeV}$-scale. In this section we discuss the relevance of three key bounds on our model: peak searches, beam dumps and non-terrestrial considerations.

An established way to find strong model independent bounds on heavy sterile neutrinos is through the study of two-body decays of mesons, particularly pions and kaons $[49,50]$. Due to the two-body kinematics, the magnitude of the neutrino mass manifests itself as a monochromatic line in the charged lepton energy spectrum at $E_{l}=\left(m_{\pi(K)}^{2}+m_{l}^{2}-m_{N}^{2}\right) / m_{\pi(K)}^{2}$. These peak searches provide strong bounds on the sterile-active mixing, while remaining agnostic as to the ultimate fate of the sterile neutrino, which may be extremely long lived. ${ }^{4}$ Meson decay peak searches have taken place for $\pi \rightarrow \nu e(\mu)$ and $K \rightarrow \nu e(\mu)$ and strongly bound active-sterile mixing angles at low masses. The strength of these bounds is not a function of sterile neutrino decay-rate, and as such, peak searches tend to perform worse at higher masses in comparison to bounds from experiments which derive their signal from large sterile neutrino decay rates.

The tightest bounds on $\mathrm{MeV}$ scale sterile neutrinos come from beam dump experiments. Beam dump experiments study the particles emitted during proton collisions with a target. Although BSM particles may be produced directly [51, 52], sterile neutrinos would predominantly arise as secondary decay products of mesons produced in the initial collision. The set-up required for such an experiment is quite minimal - a proton beam, a target and a down-wind detector - and for this reason searches of this type have taken place at many accelerator complexes, taking advantage of preexisting proton beams in their design. Seeking to produce and observe the subsequent decay of the sterile neutrinos, the sensitivity of beam dump experiments is driven by both flux intensity and the decay

\footnotetext{
${ }^{4}$ If, on the other hand, the sterile neutrino is extremely short-lived, these bounds may be weakened. If the particle decays on the scale of the experiment, it may produce a multi-lepton final state and escape observation by the single-lepton analysis cuts.
} 
rate of the heavy sterile neutrino, which typically scales as $\left(\Gamma \propto m_{N}^{3}\right) \Gamma \propto m_{N}^{5}$ for (semi-) leptonic decays. As such they typically set tighter bounds as the sterile neutrino mass increases. As discussed in the introduction, PS-191, which ran in parallel with the BEBC bubble chamber, provides the strongest limits on active-sterile mixing for masses below the kaon mass. Above this mass, a higher energy proton beam is needed to further the same strategy. This was achieved by moving from the CERN PS to the SPS proton beam in both the CHARM [27] and NA3 [53] experiments. Beam dumps are incredibly sensitive to active-sterile mixing and limits $\left|U_{e 4}\right|^{2} \leqslant 10^{-8}-10^{-9}$ were set for $m_{N} \geqslant 200 \mathrm{MeV}$.

Results from beam dump experiments are most often presented, as we did above, as upper limits on active-sterile mixing in the context of the minimal model. However, beam dump experiments actually set two bounds: there is also a lower bound on the mixing-matrix elements, where the decay rate is so large that the sterile neutrino beam attenuates en route to the detector. In the minimal model, this lower bound is often at very large values of $\left|U_{\alpha 4}\right|^{2}$, presenting consistency issues with unitarity data, and is justifiably ignored. If one considers enhanced decay rates in a non-minimal model, however, care must be taken with existing bounds as an enhanced decay rate would modify both bounds. This can reduce the applicability of certain bounds to non-minimal models. It is instructive to discuss how to scale existing bounds on the minimal model, or indeed the bounds we will present in section 4, to an extended model which has an enhancement in the decay rate for one or more channels. By comparing the flux-folded probabilities to decay inside a detector for a beam dump experiment of baseline $L$ and detector length $\lambda$, we can map the published lower bound, $\left|\widetilde{U}_{\alpha 4}\right|^{2}$, to both the new upper and lower bound on the mixing matrix element in a non-minimal model, $\left|U_{\alpha 4}\right|$. For a generic non-minimal model in which the total decay rate is scaled by a factor $A$ with respect to the minimal model, and the decay rate into the channel of interest is scaled by a factor $B$, the constraint takes the form of Lambert's equation (at leading order in $\lambda / L$ ), and the bounds on the non-minimal mixing-matrix element are given by the two real branches of the Lambert- $W$ function,

$$
\frac{\left|\widetilde{U}_{\alpha 4}\right|^{2}}{B \kappa} \mathcal{W}_{-1}\left(\exp ^{\kappa} \frac{B}{\sqrt{A}} \kappa\right) \leqslant\left|U_{\alpha 4}\right|^{2} \leqslant \frac{\left|\widetilde{U}_{\alpha 4}\right|^{2}}{B \kappa} \mathcal{W}_{0}\left(\exp ^{\kappa} \frac{B}{\sqrt{A}} \kappa\right),
$$

where $\kappa \equiv-\Gamma_{\mathrm{T}} L /(2 \gamma \beta)$ with $\Gamma_{\mathrm{T}}$ the total decay rate calculated with $\left|\widetilde{U}_{\alpha 4}\right|^{2}$. The upper bound is primarily dependent on the decay rate into the channel of interest, governed by the parameter $B$, whilst the lower bound is predominantly sensitive to the total decay rate and the parameter $A$. Physically, the upper bound is seen to depend on how many decays are produced and is sensitive to the (possibly enhanced) decay rate into that channel, while the lower bound arises when the beam attenuates due to decay before the detector, the rate of which is governed by the total decay rate.

Although the exact behaviour of the bounds for a non-minimal BSM extension are model-dependent due to correlations between $A$ and $B$, in many situations the upper bound can be significantly simplified. We consider two distinct scenarios depending on whether the enhancement affects the decay rate of the channel being observed, or another decay channel. We write the total decay rate as $\Gamma_{\mathrm{T}}=\Gamma_{\mathrm{o}}+\Gamma_{\mathrm{c}}$, where $\Gamma_{\mathrm{c}}$ denotes the channel 
whose decay products are being measured and $\Gamma_{\mathrm{o}}$ the sum of all other decay rates. In our first scenario, the only enhancement is to the channel of interest, and the total decay rate can be written as $\Gamma_{\mathrm{T}}=\Gamma_{\mathrm{o}}+B \Gamma_{\mathrm{c}}$. In this case, the upper bound from eq. (2.3) can be simplified by expanding in the published bound, ${ }^{5}\left|\widetilde{U}_{\alpha 4}\right|^{2}$. In this approximation, the new bound is seen to be a simple scaling of the old bound

$$
\left|U_{\alpha 4}\right|^{2} \leqslant \frac{\left|\widetilde{U}_{\alpha 4}\right|^{2}}{\sqrt{B}} .
$$

This follows our naive expectations: a larger decay rate produces more events and so bounds are proportionally stronger. The lower bound on $\left|U_{\alpha 4}\right|^{2}$ has no corresponding simple form, but numerically can be seen to follow a similar scaling relationship: as the enhancement goes up, the bound moves to lower values of the mixing-matrix element. In this case, apart from a replacement of the minimal $\left|\widetilde{U}_{\alpha 4}\right|^{2}$ by an effective mixing-matrix element $\left|U_{\alpha 4}\right|^{2} / \sqrt{B}$, the bounds are to a good approximation unchanged. The situation is qualitatively different in our second scenario, however. In this case, we consider an enhancement to $\Gamma_{\mathrm{o}}$, so that $\Gamma_{\mathrm{T}}=A \Gamma_{\mathrm{o}}+\Gamma_{\mathrm{c}}$. We find that the upper bound is unchanged to leading order, $\left|U_{\alpha 4}\right|^{2} \leqslant\left|\widetilde{U}_{\alpha 4}\right|^{2}$. However, the lower bound moves to smaller values as the enhancement increases. For large enhancements, this can significantly reduce the region of parameter space in which an experiment can bound the model. We will return to these simplified models of decay rate enhancement in section 4.

We note in passing that the limit of large $\lambda / L$ can also be relevant for eq. (2.3). This corresponds to experiments where production and detection occur inside the detector, which can be seen as zero baseline beam-dumps. We find that these experiments produce only an upper bound on the mixing angle, as the number of incoming sterile neutrinos can no longer be attenuated through decays occurring before the detector.

Although peak searches and beam dumps set some of the most stringent upper limits on mixing, non-terrestrial measurements may also place bounds on such long lived sterile neutrinos due to their effect on the evolution of the early universe. Heavy sterile neutrinos can have a strong impact on the success of Big-Bang Nucleosynthesis (BBN) by both speeding up the expansion of the universe with their additional energy, and thus effecting an earlier freeze out of the neutron-proton ratio, as well as potentially modifying the spectrum of active neutrinos via their subsequent decays. If, however, the sterile has sufficiently short lifetime then their effect on $\mathrm{BBN}$ is mitigated as the bulk of thermally produced sterile neutrinos have decayed long before $T_{\mathrm{BBN}} \approx 10 \mathrm{MeV}$ [54]. The strength of these bounds have been estimated conservatively for a single sterile neutrino, $m_{N}<m_{\pi^{0}}$, as $[55,56]$

$$
\begin{array}{rlrl}
\tau_{\mathrm{N}} & <1.287\left(\frac{m_{N}}{\mathrm{MeV}}\right)^{-1.828}+0.04179 \mathrm{~s} & & \text { for } U_{\mu 4} \text { or } U_{\tau 4} \text { mixing, } \\
\tau_{\mathrm{N}}<1699\left(\frac{m_{N}}{\mathrm{MeV}}\right)^{-2.652}+0.0544 \mathrm{~s} & & \text { for } U_{e 4} \text { mixing, }
\end{array}
$$

at the $90 \%$ CL. Although the scenario for $m_{N}>m_{\pi^{0}}$ has not been studied in as much detail, an often quoted bound is that $\tau_{N}>0.1 \mathrm{~s}$ is excluded under current BBN measurements [55].

\footnotetext{
${ }^{5}$ These are typically of the order $10^{-4}-10^{-8}$ and such an expansion is a very good approximation.
} 
In the minimal model, this upper bound on the sterile neutrino lifetime is directly mapped to a minimum bound on the active-sterile mixing elements $U_{\alpha 4}$. However, even a modest increase in the total sterile neutrino decay rate, for example by additional interactions in the sterile neutrino sector leading to decays that are not mixing suppressed, pushes the total sterile neutrino lifetime below $0.1 \mathrm{~s}$ and avoids these bounds, while still leaving channel specific signatures observable at SBN as the upper bounds are independent on total decay rate. Similarly in a non-standard model of the early universe, these bounds may not apply. Therefore, although setting important complementary bounds on models of sterile neutrino decay, model dependent factors make it possible for discrepancy between peak search, beam dump and cosmological constraints. As such a wide program of experimental work is desirable, with as varied a methodology as possible, to best identify new physics.

\section{Simulation of SBN}

SBN consists of three LArTPC detectors (SBND, MicroBooNE and ICARUS) located in the Booster neutrino beam. The Booster neutrino beam is a well understood beam, having been recently studied by the MiniBooNE experiment. For the purposes of this analysis each detector is assumed to be identical apart from their geometric dimensions. We simulate the event numbers and distributions at each detector site using a custom Monte Carlo program which allows efficiencies to be taken into account arising from experimental details such as energy and timing resolution in a fully correlated way between observables, and provides us with event level variables for use in a cut-based analysis. We compute the total number of accepted events in channel "c" via the following summation,

$$
N_{\mathrm{c}}=\left.\sum_{i} \frac{\mathrm{d} \phi}{\mathrm{d} E}\right|_{E_{i}} P_{\mathrm{D}}\left(E_{i}\right) W_{\mathrm{c}}\left(E_{i}\right)
$$

where $P_{\mathrm{D}}(E)$ is the probability for a sterile neutrino of energy $E_{i}$ to travel the baseline distance and then decay inside the detector labelled $\mathrm{D}$. The simplest approximation is to ignore all geometric effects, so that every particle travels exactly along the direction of the beam line, which gives the following probability

$$
P_{\mathrm{D}}(E)=e^{-\frac{\Gamma_{\mathrm{T}} L}{\gamma \beta}}\left(1-e^{-\frac{\Gamma_{\mathrm{T}} \lambda}{\gamma \beta}}\right) \frac{\Gamma_{\mathrm{c}}}{\Gamma_{\mathrm{T}}}
$$

where $\Gamma_{\mathrm{T}}\left(\Gamma_{\mathrm{c}}\right)$ denotes the rest-frame total decay width (decay width into channel c), and $L(\lambda)$ the distance to (width of) the detector. The combination $\gamma \beta$ is the usual special relativistic function of velocities of the parent particle and provides the sole dependence on energy and sterile neutrino mass $m_{N}$ of the expression

$$
\frac{1}{\gamma \beta} \equiv \frac{m_{N}}{\sqrt{E^{2}-m_{N}^{2}}} .
$$



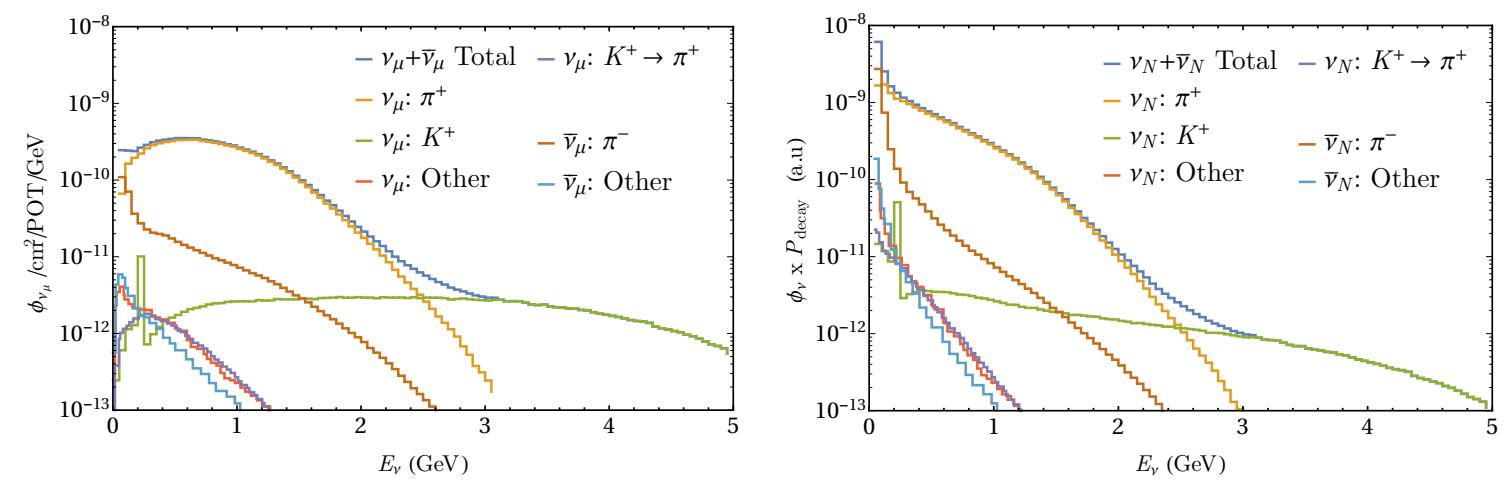

Figure 2. Left: the composition of fluxes of $\nu_{\mu}$ and $\bar{\nu}_{\mu}$ at MicroBooNE with horn in positive polarity (neutrino mode). "Other" refers to contributions primarily from meson decay chains initiated by meson-nucleus interactions. Right: fluxes weighted by the probability to decay inside MicroBooNE, for a sample $25 \mathrm{MeV}$ sterile neutrino with equal $\left|U_{e 4}\right|^{2}=\left|U_{\mu 4}\right|^{2}$. Requiring that the sterile neutrino decays inside the detector has the effect of vastly increasing the importance of lower energy bins, where traditionally cross-section induced background effects are small.

As we are exploring a large parameter space, often this expression takes a simplified form depending on the size of $\Gamma_{\mathrm{T}} \lambda / \gamma \beta$ :

$$
\begin{array}{ll}
\Gamma_{\mathrm{T}} \lambda \ll 1 & P_{\mathrm{D}}=e^{-\frac{\Gamma_{\mathrm{T}} L}{\gamma \beta}} \frac{\Gamma_{\mathrm{c}} \lambda}{\gamma \beta}+\mathcal{O}\left(\Gamma_{\mathrm{T}}^{2} \lambda^{2}\right), \\
\Gamma_{\mathrm{T}} \lambda \gg 1 & P_{\mathrm{D}}=e^{-\frac{\Gamma_{\mathrm{T}} L}{\gamma \beta}} \frac{\Gamma_{\mathrm{c}}}{\Gamma_{\mathrm{T}}}+\mathcal{O}\left(\frac{1}{\Gamma_{\mathrm{T}} \lambda}\right),
\end{array}
$$

where the rate for slowly decaying particles can be seen to grow with detector size until a width of $\lambda \sim \gamma \beta \Gamma_{\mathrm{T}}^{-1}$. For detectors longer than this scale, the event rate becomes independent of detector size, as most sterile neutrinos decay within a few decay lengths.

The spectral flux of sterile neutrinos impinging on a SBN detector, $\mathrm{d} \phi / \mathrm{d} E$, is estimated as described in section 2.1. Of crucial importance to this is accurate knowledge of active neutrino fluxes at all three SBN detectors. These are calculated from published MiniBooNE fluxes [35], after scaling by appropriate $1 / r^{2}$ baseline dependence, e.g. $(470 / 540)^{2} \approx 1.3$ at MicroBooNE. This is similarly scaled by $1 / r^{2}$ for ICARUS at $600 \mathrm{~m}$, however, an additional energy dependent flux modifier is applied for SBND at 110m to account for the softer energy spectrum due to the proximity of the detector to the production target [14]. We consider sources of neutrinos that are relevant including wrong sign neutrinos, smaller sub-dominant $K^{+} \rightarrow \pi^{+} \rightarrow \nu_{\alpha}$ sources as well as other contributions, predominantly from meson decay chains initiated by meson-nucleus interactions. The neutrino spectrum at MicroBooNE is shown in the left panel of figure 2. In the right panel, we also show the effective spectrum of decaying particles at MicroBooNE. As the decay probability for any given sterile neutrino scales as $1 /\left|P_{N}\right|$, we see an enhancement of the lowest energy parts of the spectrum. This is in stark contrast to standard neutrino interaction cross sections, which tend to scale as $E_{\nu}$. This low-energy bias exaggerates the kinematic differences between our decay-in-flight signal and the dominant background events. 
Finally, the function $W_{\mathrm{c}}(E)$ is a weighting factor which accounts for all effects which reduce the number of events in the sample: for example, analysis cuts or detector performance effects. To compute these factors, we run a Monte Carlo simulation of the decays for a large number of sample events with a given energy. Each sterile neutrino event is associated with a decay of type c. We then apply experimental analysis cuts to the decays based on our assumptions about the detector's capabilities and backgrounds, to produce a spectrum representing the final event sample when considering events in the bucket timing window (see appendix B for details of the background analysis). The percentage of accepted events defines the weight factor for that energy. In this manner the full spectral shape of the signal is included in the total rate analysis. As a consistency check of our methodology, we also reproduce in appendix C some of the published bounds of PS-191.

\subsection{Background reduction}

In order to estimate the impact of potential backgrounds we have performed a Monte-Carlo analysis using the neutrino event generator GENIE [57]. This provides generator level information about the kinematics of the beam-driven backgrounds, with rates normalised off expected $\mathrm{NC}$ and $\mathrm{CC}$ inclusive values as published in the SBN proposal. Energy and angular smearing is then implemented to allow for approximate estimates of the effects of detector performance to the level necessary for this analysis, without the need for a full GEANT detector simulation. Energies are smeared according to a Gaussian distribution around their true $\mathrm{MC}$ energies, with a relative variance $\left.\sigma_{E} / E=\xi / \sqrt{(} E\right)$, where $\xi$ is a detector dependent resolution. For this study we take the energy resolution for EM showers, muons and protons to be $15 \%, 6 \%$ and a conservative $15 \%$ respectively, alongside an angular resolution in LAr of $1.73^{\circ}$ [14].

Of utmost importance in all studied channels is the identification of a scattering vertex, which cleanly indicates that the process is not a decay-in-flight event. Any hadronic activity localised at the beginning of the lepton track is a smoking gun for a deep-inelastic or quasielastic beam-related scattering event. Therefore we reject any event containing one or more reconstructed protons or additional hadrons. For counting this proton multiplicity we assume a detection threshold of $21 \mathrm{MeV}$ on proton kinetic energy in liquid Argon [58], after smearing. Background events with energies below this threshold and events that do not contain any protons (such as events originating from coherent pion production) remain a viable background and further rejection must come from the kinematics of the final state particles only. The kinematics of such daughter particles originating from decay-inflight and backgrounds from scattering events, however, have strikingly different behaviour leading to strong suppression capabilities.

As a representative example of our analysis we discuss the backgrounds associated with the decay $N \rightarrow \mu^{ \pm} \pi^{\mp}$, the channel with largest expected beam related backgrounds in all SBN detectors, the dominant component of which arises from genuine charged current $\pi \mu$ production. These events can be produced incoherently, often with large hadronic activity and so will greatly be reduced by the cut on a scattering vertex, or from coherent scattering, where the neutrino scatters from the whole nucleus. Coherent cross-sections for these processes have been studied in MiniBooNE [59], MINER $\nu \mathrm{A}$ [60] and lately T2K and cross- 


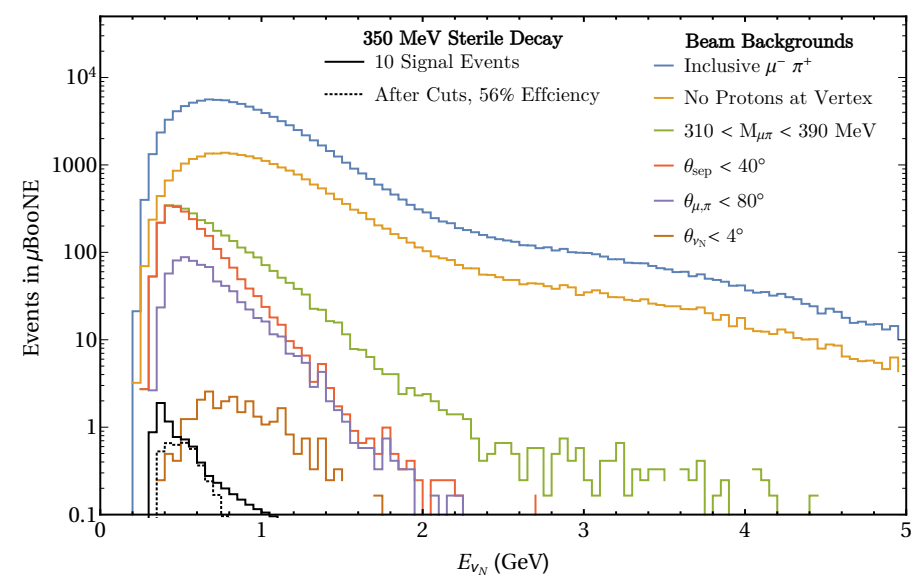

Figure 3. Reconstructed sterile neutrino energy spectra for $\mathrm{CC} \nu_{\mu}$ backgrounds in comparison to a $350 \mathrm{MeV}$ decaying sterile neutrino at MicroBooNE, normalised to 10 signal events. Total expected background of 98,013 events is reduced to $\approx 27$ by successive kinematic cuts (as listed in legend) which utilise the stark differences between decay-in-flight and scattering kinematics. Further cuts on energy would allow for even greater reduction.

sections appear to agree with Monte Carlo calculations based on the Rein-Sehgal model [61] and generally do not have an additional hadronic component to cut on. Kinematics of the daughter particle alone but be used for background rejection. There has been a noted deficit of forward going muons [61] in these coherent cross-sections, which is in stark comparison to the relatively forward behaviour of sterile neutrino decays.

Furthermore, this channel, and indeed $e^{ \pm} \pi^{\mp}$, has a powerful discriminator in the reconstructed invariant mass of the charged particle pair, e.g. $M_{l^{ \pm} \pi^{\mp}}^{2}=m_{l}^{2}+m_{\pi^{ \pm}}^{2}+2\left(E_{l} E_{\pi}-\right.$ $\left|P_{l}\right|\left|P_{\pi}\right| \cos \theta_{\text {sep }}$ ) for $N \rightarrow \pi^{ \pm} l^{\mp}$, which sum to that of the the parent sterile neutrino (within detector resolution), whereas the background forms a broad spectrum across the energies of the incoming neutrinos. On top of this strong invariant mass discriminator, these two body decays allow for reconstruction of the parent sterile neutrino angle with respect to the beamline which is very close to on-axis, as opposed to the more isotropic backgrounds. We find that approximately $95 \%$ of the reconstructed sterile neutrino angles from these decays are inside a $4^{\circ}$ cone centred on the beamline.

We show the effect of our cuts for this channel in figure 3, which ultimately leads to a reduction in the inclusive $\mu \pi$ event rate at SBND (MicroBooNEand ICARUS) from 1,530,900 (98,013 and 164,716) to 323 (27 and 46) while maintaining a signal efficiency of $56 \%$. This level of background suppression crucially relies on the angular and energy resolution of LAr detectors, but requires no modification to the current design.

For a parallel discussion of the backgrounds for the remaining channels see appendix B.

\subsection{Role of event timing}

On top of the impressive background rejection capabilities of LAr from kinematic cuts, there is the potential for an even greater background suppression by considering the time of arrival of observed events. Although the drift time of electrons in LAr can be as large as 
$100 \mu \mathrm{s}$, the ionisation and excitation of Argon from the passage of a charged particle also produces $128 \mathrm{~nm}$ scintillation light of which there is a nano-second scale contribution from the decay of the excited state $\mathrm{Ar}_{2}^{*}$ [62]. LAr is transparent to this light, and if the light detection system (LDS) employed by the SBN detectors has a nanosecond resolution, this can allow for precise timing to be attached to each TPC triggered event.

Light neutrinos propagate and reach the furthest detector of the SBN complex after approximately $2 \mu \mathrm{s}$. In the conventional physics program of the SBN, the timing of these events play an important role in the analysis of backgrounds, tight timing windows are placed around the $19.2 \mu$ s beam spill to limit constant rate backgrounds such as cosmogenic events [14]. The LDS of both SBND and ICARUS, however, are potentially able to achieve significantly better timing resolution than this, around 1-2 ns depending on the exact technology used, which potentially allows for the use of both bucket and spill structure in the background analysis. The BNB consists of 81 Radio-Frequency buckets of approximately $2 \mathrm{~ns}$ length, separated by $19 \mathrm{~ns}$, to form the $19.2 \mu$ s spill with a frequency of $3 \mathrm{~Hz}$ [14]. If this nano-second resolution is indeed achieved, it allows for events in individual buckets to be identified. Such a nano-second resolution was achieved previously by the PMTs used in MiniBooNE [14], with potential for improvement in the next generation SBN detectors.

As particles with finite rest mass, heavy sterile neutrinos will propagate at subluminal speeds which can produce observable timing delays. This effect begins to become relevant when the sterile neutrinos have MeV-scale masses and above. As the flux of decaying sterile neutrinos is inversely proportional to its momentum after convolving with their decay probability, many of these low energy sterile neutrinos are travelling at sufficiently slower speeds than their light counterparts to be distinguishable. Shown on the right of figure 4 is the fraction of events that are expected to fall outside the bucket window in both SBND and ICARUS. For the purposes of this study we define the beam-correlated window to be a 6 ns period, 2 ns each side of the 2 ns beam bucket. The exact width of the beam bucket window can be modified if studying channels with low expected backgrounds. In this section, we consider only the timing of events relative to the bucket window, ${ }^{6}$ a structure which repeats every $21 \mathrm{~ns}$. Delayed events can be observed in any subsequent window, producing a 21-fold degeneracy in their reconstructed arrival time. This lends a cyclical nature to the timing information, with a distinctive structure at the different detector sites for larger masses. Some illustrative timing distributions are shown on the left of figure 4 for a 25 and $350 \mathrm{MeV}$ sterile neutrino.

We find a significant proportion of sterile neutrino events distributed throughout the inter-bucket region. Events which fall into the beam-bucket timing window have to be analysed on top of all known beam-related backgrounds, but events in the inter-bucket window have significantly reduced beam-correlated backgrounds. For larger masses, we have shown that the majority of events fall into these regions, and this may allow for a low background search strategy for decaying sterile neutrinos. Instead of beam-correlated

\footnotetext{
${ }^{6}$ Absolute arrival times could in principle be used, but this would require good synchronisation between geographically separated clocks. Alternatively, the relative timing between signal and beam-related backgrounds could be used. However, we do not consider these options further.
} 

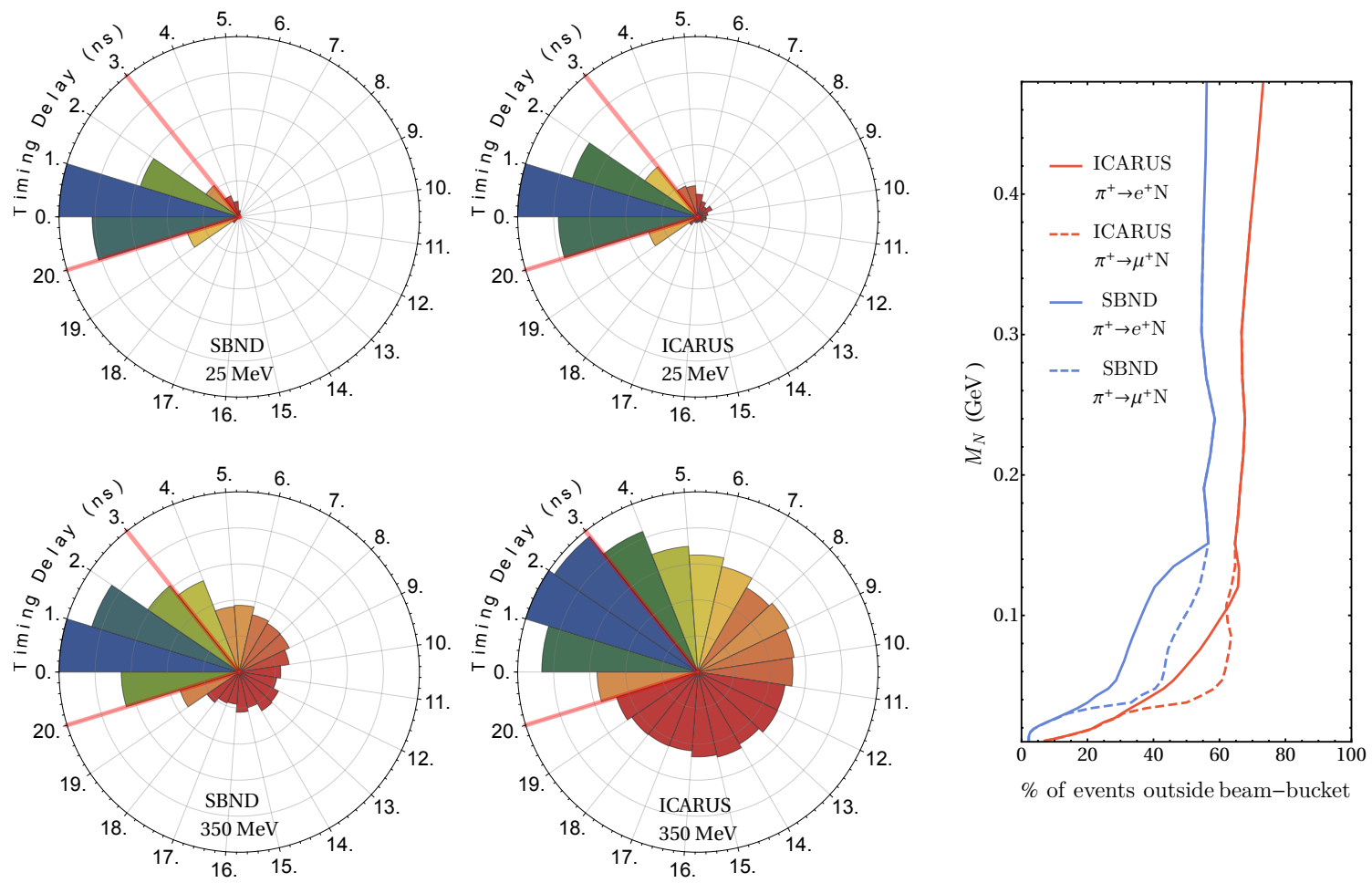

Figure 4. Left: the timing delay of sterile neutrino decays in nano-seconds for both a $25 \mathrm{MeV}$ (top) and $350 \mathrm{MeV}$ (bottom) sterile neutrino at the SBND and and Icarus detectors (110 and 600m respectively). A 4 ns beam bucket window is shown highlighted in red from 0 to 4 ns, followed by an additional $17 \mathrm{~ns}$ gap. The timings are calculated as a difference to the time of flight of a active neutrino, assuming the decay occurred in a uniform sample across the $50 \mathrm{~m}$ BNB decay pipe. A timing resolution of $1 \mathrm{~ns}$ is assumed to smear the observed events. Right: the percentage of sterile neutrino decay events that fall into the inter-bucket region as a function of sterile neutrino mass for SBND and ICARUS, assuming a flux derived from $U_{e 4}\left(U_{\mu 4}\right)$ mixing in solid (dashed) lines. Both SBND and ICARUS see a sizeable fraction of total events outside the beam bucket windows when the sterile neutrino mass exceeds $\approx 10 \mathrm{MeV}$.

backgrounds, the constant rate backgrounds will limit the sensitivity for this analysis. Understanding these backgrounds in detail is beyond the scope of this work; however, we expect the strongly forward kinematics, combined with in situ beam-off measurements will allow for a very low backgrounds to be obtained.

In the following sections, timing information will inform our work in three ways. First we will compute SBN's sensitivity to decaying sterile neutrinos assuming the full backgrounds, reduced only by the cut-based analysis discussed previously. This is a proven sensitivity, applicable for all sterile neutrino masses and detectors and is independent of the attainable timing resolution. Secondly, we compute a backgroundless sensitivity. This can be seen as either the result of improved analysis techniques, or as the inclusion of timing information at SBND and ICARUS for the largest masses. Finally, in section 3.2, we will study the use of the timing information itself to constrain the underlying model of decaying sterile neutrinos. 


\subsection{Event spectra}

The differential distributions from heavy sterile neutrino decay tend to produce distinctive low-energy distributions of events with an appreciably forward direction. The tendency towards low energies is predominantly due to the higher decay rates of low-energy particles, which leads to factors of $1 / E_{\nu}$ in the event rate formula eq. (3.1). The forward trajectory is inherited from the kinematics of a boosted object decaying in flight. However, this effect is slightly mitigated by the preference for lower energy decays, meaning that lower energy sterile neutrinos are more likely to decay, which are the least boosted objects.

We show an example of a distribution for electron-positron production in the top left panel of figure 5. For the lowest masses that we consider, almost all events have energies below $0.5 \mathrm{GeV}$, in this case illustrated by the blue histogram. The distribution tends towards larger energies as the mass of the sterile neutrino increases, but for sterile neutrino masses less than the kaon mass, never produces significant numbers of events above $1 \mathrm{GeV}$. As we can see in figure 5, the number of events in the lowest energy bin is strongly indicative of the mass of the parent particle, and therefore the lowest energy events will play a strong role in model discrimination. However, in the cut-based analysis which we outline in appendix B the lowest energy event distribution is significantly reduced due to poor efficiency's at low-energy in our cuts, as can be seen in the top right panel of figure 5 . In figure 6 we show the same distributions for electron-pion production, noting similar spectral features of the electron-positron channel. Indeed this behaviour qualitatively exists in all channels studied. In the inset of figure 6 , we highlight the differences that an accurate timing resolution can give, with the in-bucket and out of bucket spectra showing very significant differences. Through optimisation of this part of the analysis, we expect the sensitivity to these models can be improved; however, this is beyond the scope of the present work. The angular spectrum is expected to be very informative in these models, and the events are predict to align with the beam direction. The red histogram in the lower panel of figure 5 illustrates an expected distribution for the four momentum of a $e^{+} e^{-}$pair in the decay $N \rightarrow \nu e^{+} e^{-}$. We compare it to the expected distribution found for events without the low-energy biasing effect of decay-in-flight, with an unphysical energy independent decay rate (denoted 'flat', shown in blue). Not only does the decay-in-flight probability lead to a lower energy events, but it also makes the angular distribution less forward.

\section{Results}

In this section, we present the results of our simulation for two analyses. In the first, we compute exclusion contours which could be expected to be set by SBN if no signal is seen. We compute these for all decay modes presented in figure 1. Our second analysis considers the phenomenological potential of energy and timing spectral information at the SBN experiment if a potential signal is observed.

Due to its proximity to the BNB target, SBND provides the majority of the statistics, and hence the sensitivity, to sterile neutrino decays. The addition of MicroBooNE and ICARUS increases the event rate by approximately $6 \%$. However, the power of the three detector SBN setup arises not from the increased statistics, but rather from the additional phenomenology of a multi-baseline experiment. We show below that ICARUS, being the 

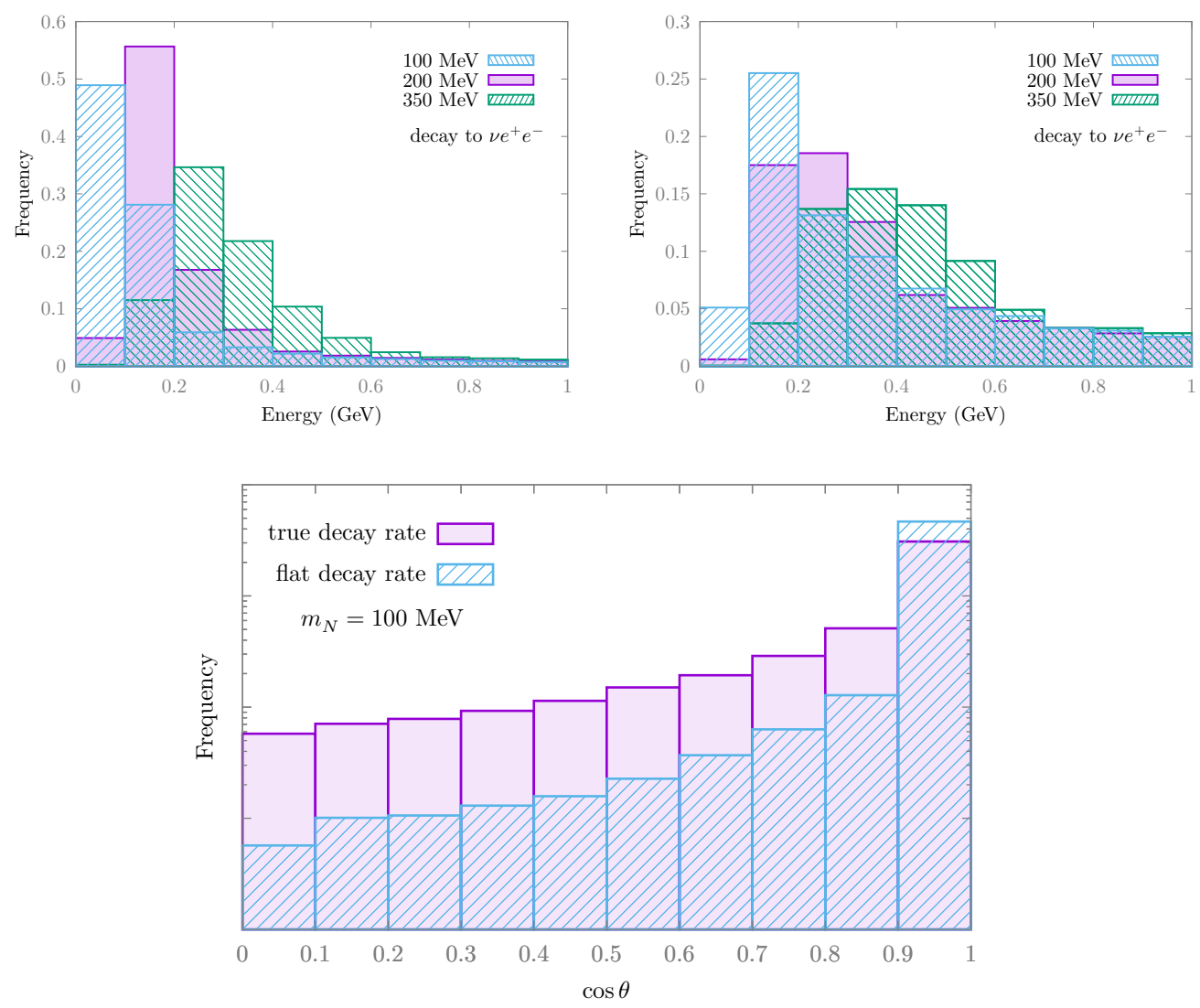

Figure 5. Top: characteristic spectra for the total energy of observed $e^{+} e^{-}$pairs seen at MicroBooNE produced in the $N \rightarrow \nu e^{+} e^{-}$decay mode, for three representative masses. In the left panel, the spectra have no analysis cuts or detector reconstruction effects applied, while on the right these are included, reducing the number of lowest-energy events. Bottom: the expected angular distributions for the $e^{+} e^{-}$pair from a sterile neutrino of mass $m_{N}=100 \mathrm{MeV}$. The red histogram shows the true expected distribution, while the blue histogram shows the distribution if we do not take into account the preferential decay rate for lower energy particles, instead using an energy independent decay rate.

furthest detector, can play an important role in study of any observed signals through precision timing measurements. Similarly, although MicroBooNE contributes a small fraction to the raw number of sterile neutrino events expected, the MicroBooNE experiment is significantly more advanced than its two SBN counterparts. At the time of writing, MicroBooNE has already collected close to $50 \%$ of its planned POT (around $3.5 \times 10^{20} \mathrm{POT}$ ) and has already presented its first results on $\nu_{\mu} \mathrm{CC}$ inclusive and $\nu_{\mu} \mathrm{CC} \pi^{0}$ interactions [63]. As such, MicroBooNE is in a unique position in that it has the potential to observe any excess in advance of SBND or ICARUS and thus to inform a possible search using the full SBN programme. Non-observation of any excess at MicroBooNE would not negatively effect the subsequent search for new physics at SBND or ICARUS significantly, however, as a large fraction of the testable parameter space is only accessible through the enhanced exposures of the full SBN programme. 


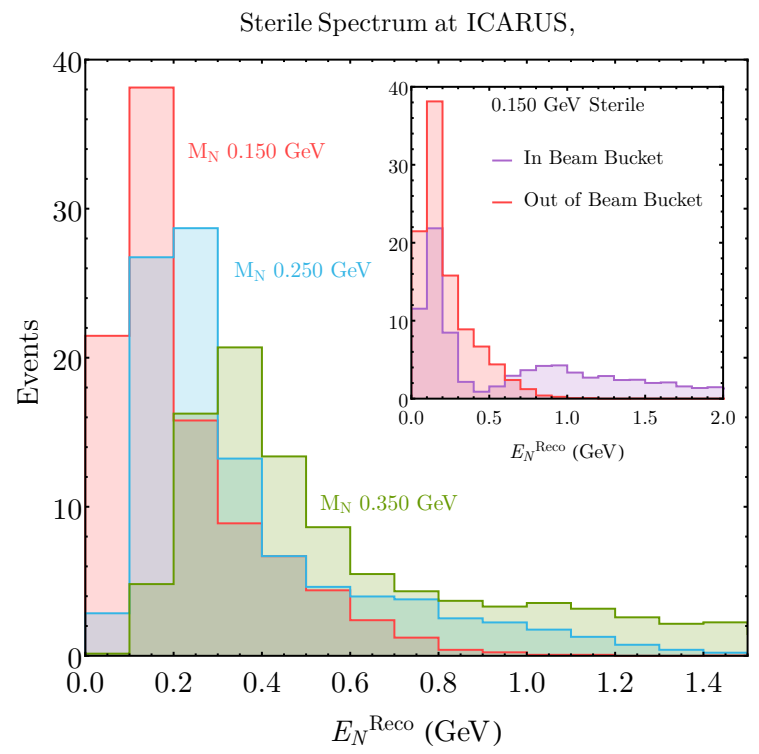

Figure 6. Characteristic spectra for the reconstructed neutrino energy for $N \rightarrow e^{ \pm} \pi^{\mp}$ and a sterile neutrino masses of 150,250 and $350 \mathrm{MeV}$. The insert shows the stark differences in spectrum when one considers events falling within the beam bucket window and without.

\subsection{Limits on sterile neutrino mixing}

We have computed the bounds that SBN could place on sterile neutrino mixing-matrix elements for all kinematically accessible visible decays. Figure 7 presents the results of our analysis assuming a combined $6.6 \times 10^{20}$ POT at SBND and ICARUS, and $13.2 \times 10^{20}$ POT at MicroBooNE. We plot the predicted upper limits on sterile neutrino mixing for the leptonic decay channels $N \rightarrow \nu_{\alpha} e^{+} e^{-}, N \rightarrow \nu_{\alpha} \mu^{+} \mu^{-}$and $N \rightarrow \nu_{\alpha} \mu^{ \pm} e^{\mp}$ as well as the semileptonic and photonic channels $N \rightarrow l^{\mp} \pi^{ \pm}, N \rightarrow \nu_{\alpha} \pi^{0}$ and $N \rightarrow \nu_{\alpha} \gamma$. The plot on the right (left) assumes that the mixing-matrix element with the electron (muon) flavour is dominant. The $y$-axis refers to a single mixing element, $\left|U_{\alpha 4}\right|^{2}$, but each bound is equally applicable to the combination $\left|U_{\alpha 4}\right|\left|U_{\tau 4}\right|$, as although production must proceed through electron-neutrino or muon-neutrino mixing, the decay can take place through $U_{\tau 4}$ driven processes. The lower solid coloured lines are the background-less 90\% CL upper limit contours defined as 2.44 events following the procedure of ref. [66]. This represents the best expected sensitivity at the SBN program, assuming perfect signal efficiency. We also present the results of the cut based background analysis discussed in section 3.1 (upper solid coloured lines). Depending on the optimisation of the analysis, including the possibility of using improved timing information, we expect the ultimate sensitivity to be within the solid-shaded region, lying between the proven cut-based sensitivity and the backgroundless one.

The increased event rates at SBN compared to those of PS-191 allows for an improvement of the bounds on $\left|U_{e 4}\right|^{2}$ and $\left|U_{\mu 4}\right|^{2}$ in all channels studied over wide regions of parameter space. The strongest bounds come from the semi-leptonic $N \rightarrow l^{ \pm} \pi^{\mp}$ searches, where mixing-matrix elements greater than $\left|U_{e 4}\right|^{2} \leqslant 10^{-9}$ can be excluded at the 90\% CL for $m_{N} \approx 0.350 \mathrm{GeV}$. The bounds have the potential to improve upon the $\pi^{-}$peak search 

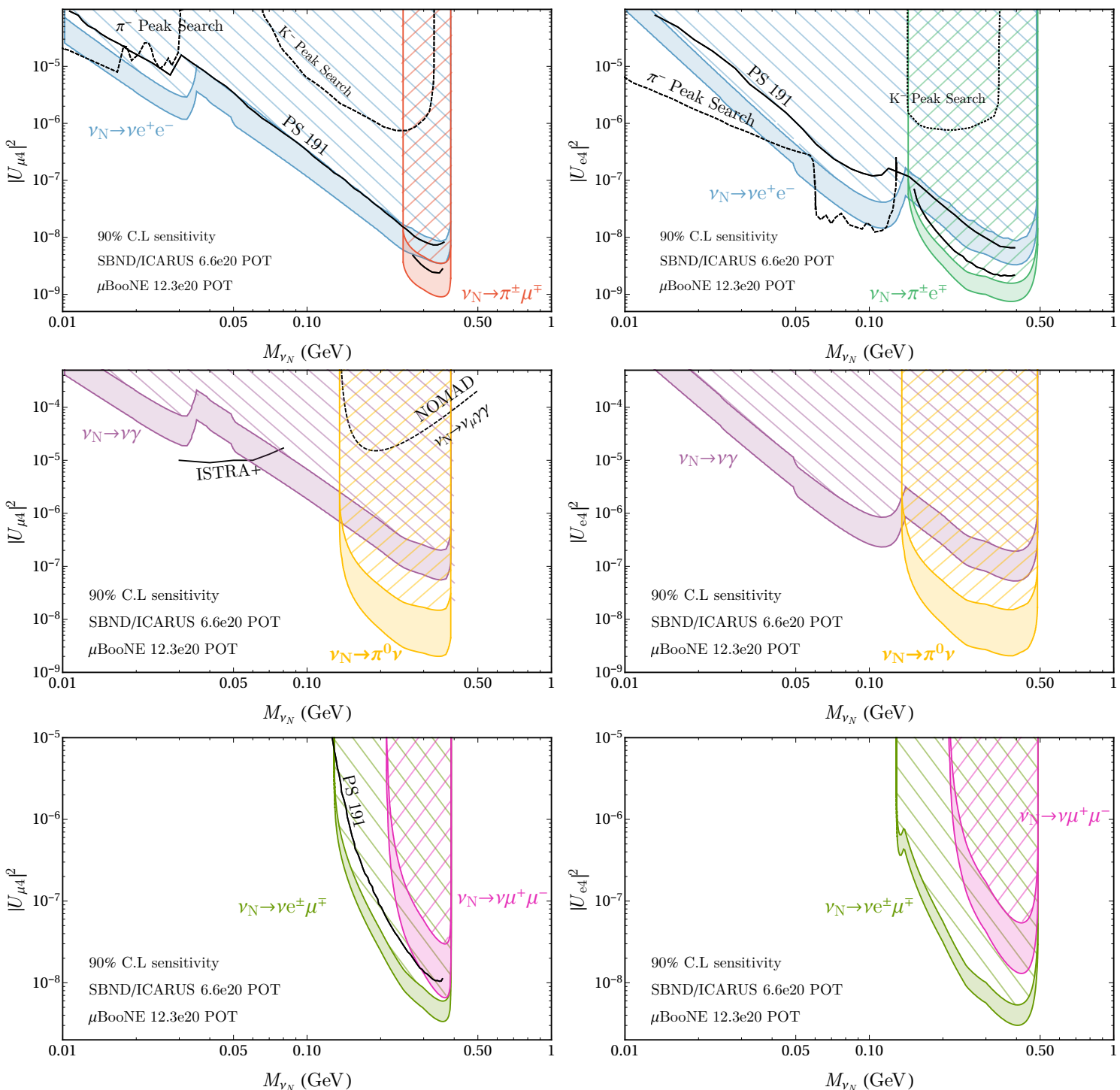

Figure 7. The predicted 90\% CL upper limit contours for the combined SBN detectors. Shown also in black solid lines is the prior best bounds from PS-191, scaled to show bounds on the minimal extension as discussed here, as well as bounds from lepton peak searches in pion and kaon decay $[49,50]$ (dashed black lines), although Kaon peak searches are not competitive in the minimal model. Note that the peak searches are only valid when bounding pure mixing combinations, e.g. $\left|U_{\mu 4}\right|^{2}$ and not $\left|U_{\mu 4}\right|\left|U_{\tau 4}\right|$. The photonic channels have little or no direct bounds, with ISTRA+ bounding the radiative decay [64] and reinterpreted $N \rightarrow \nu \gamma \gamma$ bounds at NOMAD on $N \rightarrow \nu \pi^{0}$ [65]. In all panels, the mixing matrix elements not shown on the $y$-axis are zero. 
bounds for $m_{N} \leqslant 0.033 \mathrm{GeV}$ and $m_{N} \leqslant 0.138 \mathrm{GeV}$ for muon and electron mixing respectively, if the backgrounds can be further suppressed, possibly through the use of timing information.

Additionally, we show that the previously poorly bounded photonic-like channels $N \rightarrow$ $\nu_{\alpha} \pi^{0}$ and $N \rightarrow \nu_{\alpha} \gamma$ can be probed across the entire parameter space, providing new constraints on exotic sterile neutrino signatures. The potential beam-related backgrounds are large for these photonic channels, the effect of which is a much wider separation between our cut-based limits and the optimal ones. These photonic channels allow SBN to probe the electromagnetic nature of the sterile neutrino, placing bounds on any models containing enhanced couplings to photons. For sterile neutrinos whose mass lies $m_{\pi}^{0} \leqslant m_{N} \leqslant m_{\pi}^{ \pm}+m_{\mu}$ and mix primarily with muons, the $N \rightarrow \nu_{\mu} \pi^{0}$ channel can extend the limits beyond that of the traditional $e^{+} e^{-}$searches to probe new parameter space, even in the purely minimal model. For sizeable $U_{e 4}$, the $\pi^{0}$ bounds are less powerful than that of the semi-leptonic $N \rightarrow e^{ \pm} \pi^{\mp}$ when one assumes the minimal model.

Although we have plotted the limits on mixing angles in figure 7 in terms of the parameters of the minimal model, they are model independent in the sense that an enhanced decay rate in that channel would only alter the interpretation of the $y$-axis. If the enhancements to the decay rates are modest, to reinterpret any bounds on figure 7 in the context of a non-minimal extension in which the channel of interest is enhanced by $(1+\alpha)$ then the quantity bounded on each vertical axis is given approximately by $\left|U_{\alpha 4}\right|^{2} / \sqrt{1+\alpha}$ as discussed in section 2.3. However, for larger enhancements, the lower-bound on the mixing-matrix element must also be considered. In figure 7, this bound lies at large values of $|U|^{2}$, and is not shown in the plots, but it is also affected by an enhanced decay rate and can become relevant of reasonable enhancements. This can be seen in the left panel of figure 8 , where we show the region of parameter space that SBN could exclude when studying the decay mode $N \rightarrow \nu e^{+} e^{-}$as we increase its decay rate by factors of $10,10^{2}, 10^{3}$ and $10^{4}$. As was shown analytically in section 2.3 , the upper bound scales as $1 / \sqrt{1+\alpha}$ as the number of events in the detector increases. However, the enhancement eventually leads to significant beam attenuation before the detector. This alters the lower bound, which begins to move to smaller values of the mixing-matrix element, opening up a region of parameter space in the top-right of the plot. In the right panel of figure 8, we show an alternative non-minimal model in which the decay rate $\Gamma\left(N \rightarrow \nu e^{+} e^{-}\right)$is held constant, but the total decay rate is enhanced. This could be due to the enhancement of a decay to visible or invisible final states. In this scenario, the upper bound remains unchanged as the rate is enhanced (to leading order in $\lambda / L$ ), but the enhanced total decay rate leads to beam attenuation and fewer sterile neutrinos reach the detector. Eventually, the lower bound is reduced significantly, and the experiment loses sensitivity over much of the parameter space of the minimal-model. Although an enhanced total decay rate could produce a larger visible signal in another channel, or indeed in another experiment, if the decay is predominantly to three neutrinos or dark sector particles many existing bounds may not apply. We note that enhancements on the scale of $\alpha=10^{4}$ could be expected if the novel decay proceeds without mixing suppression. Every bound presented in figure 8 can be reinterpreted in terms of these non-minimal models using the scalings as discussed 

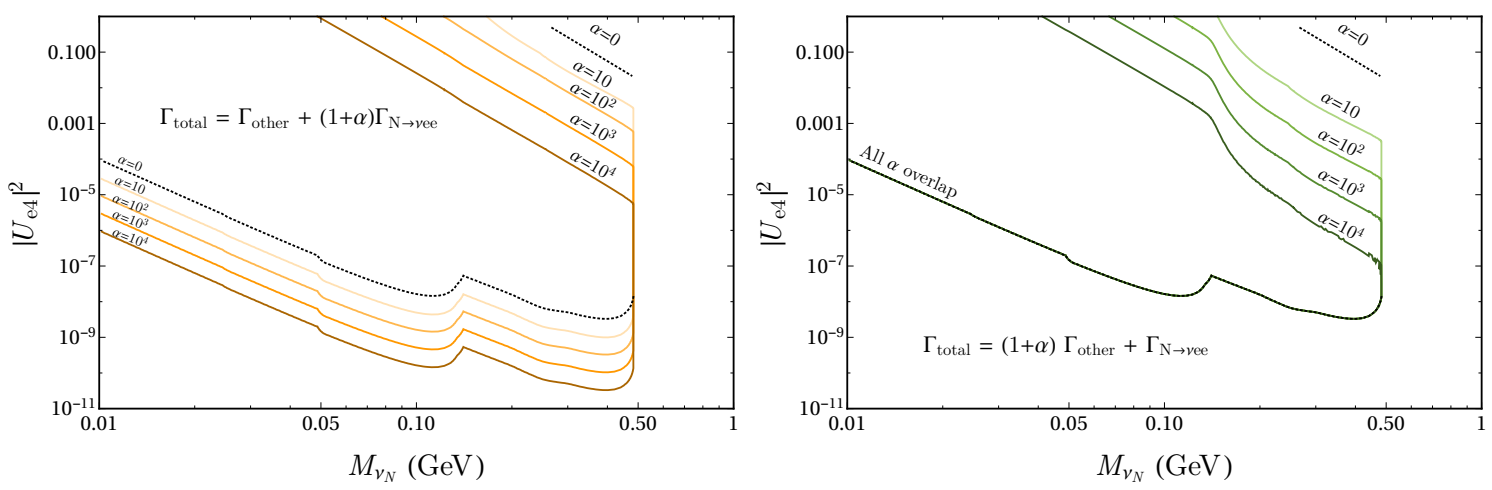

Figure 8. The 90\% CL contours for the decay $N \rightarrow \nu e^{+} e^{-}$assuming dominant $U_{e 4}$ mixing in non-minimal scenarios, at leading order in $\lambda / L$. On the left, we enhance the rate of the $e^{+} e^{-}$ channel itself by factors shown in the labels while keeping all other decay rates constant. The excluded region remains roughly constant but shifts downwards. On the right, we show the effects of keeping the $N \rightarrow \nu e^{+} e^{-}$decay rate constant, but enhancing the total decay rate. The sensitive region shrinks quickly as $\alpha$ increases, allowing non-minimal models to escape detection.

in section 2.3, and highlights why searching across the whole parameter space is necessary in all kinematically allowed decay channels.

\subsection{Timing information to study an observed signal}

In addition to being able to reduce beam-related backgrounds, a precise knowledge of the timing of any observed events can also be used to discriminate between potential models and aid parameter estimation. If a potential signal is observed, it would be highly desirable to establish whether the excess is associated with a heavy particle travelling from source to detector. An analysis based on the energy spectrum alone would struggle with this determination — we could not discount mis-understood beam-related backgrounds, unknown nuclear effects, or other models that mimic the low-energy spectrum. The angular distribution of events would also be highly informative, we have seen that heavy particle decays are likely to be associated with collimated decay products, but this would be only indirect evidence of a heavy particle, and could be associated with other models. For example, active neutrino scattering via a light mediator could also mimic this behaviour. However, as all beam-related backgrounds will be correlated with the Booster proton buckets, the observation of events with times outside of the BNB beam bucket window (and travelling in a forward direction) would be a smoking gun signal of a sub-luminal propagating parent.

We estimate the required timing resolution by simulating the distribution of arrival times for a given sterile neutrino mass. We then compute the compatibility of this data with a beam-bucket hypothesis, where all event timing is consistent (within errors) with being within the beam-bucket. We only study the shapes of these timing distributions, allowing the normalization to float, and in this sense the beam-bucket hypothesis encompasses all sources of particles which would appear beam-correlated. The beam-bucket hypothesis is defined as the assumption that all events originate in a 6 ns window surrounding the BNB beam spill, smeared by a Gaussian with a width of the assumed time resolution. We define 

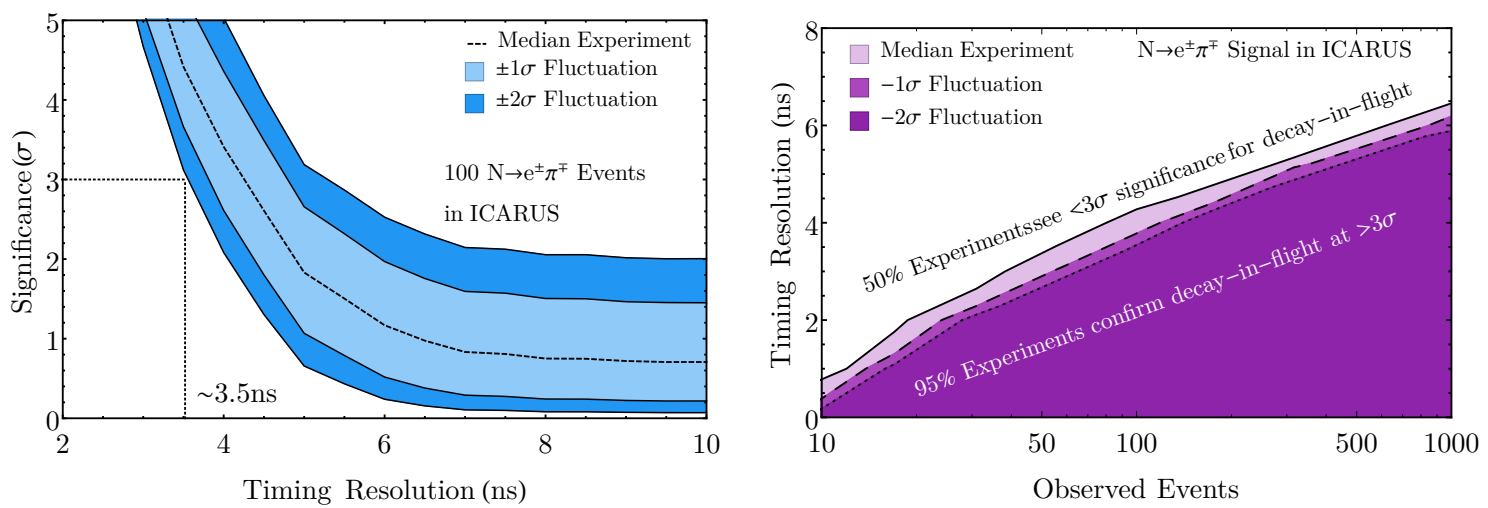

Figure 9. Left: expected significance at which ICARUS can exclude a beam-correlated origin from timing information alone, as a function of assumed timing resolution. This assumes a hypothetical signal of $100 e^{ \pm} \pi^{\mp}$ events consistent with $N \rightarrow e^{ \pm} \pi^{\mp}$. Right: as the number of observed events goes down, it is significantly harder to establish a time delay. We show the timing resolution required for a given number of events for the median experiment (solid line) as well as for $1 \sigma$ (dashed line) and $2 \sigma$ (dotted line) downward fluctuations.

our test statistic as [67]

$$
t_{m}=-2 \ln (\mathcal{L})=2 \sum_{i=1}^{N}\left\{\mu_{i}(m)-n_{i}+n_{i} \ln \left[\frac{n_{i}}{\mu_{i}(m)}\right]\right\}
$$

where $\mu_{i}(m)$ is the expected number of events in bin $i$ if the sterile neutrino is of mass $m$. Using this statistic we have run a binned Maximum Likelihood analysis of the reconstructed time of arrival $\Delta T$, assuming events are Poisson distributed. We compute the distribution for $t_{m}$ by Monte Carlo to ensure that we account for all non-gaussianity in the likelihood function.

As the timing is solely a function of the initial sterile neutrino energy and mass, these results hold for all channels studied. Without loss of generality, we restrict our discussion to the semi-leptonic channel $N \rightarrow e^{ \pm} \pi^{\mp}$. On the left panel of figure 9, we show the timing resolution required to exclude the beam-bucket hypothesis at a given statistical significance. This plot assumes that ICARUS has observed an excess of 100 events due to a $300 \mathrm{MeV}$ sterile neutrino. To guarantee that ICARUS can reject the beam-bucket hypothesis at at least $3 \sigma$ significance in $95 \%$ of pseudo-experiments, we require a timing resolution of $\leqslant 3.5$ ns. As the number of observed events increases, the timing resolution required to rule out a beam-correlated origin decreases, as we show in the right panel of figure 9 .

Although establishing that a signal arrived outside of the beam window would be an exciting sign of new physics, it would not necessarily establish a heavy propagating parent. For example, if an unaccounted for process had a fixed time delay with respect to the neutrino beam, $\Delta_{t}$, such as the relaxation time of an excited atom, it could produce events in the inter-bucket region for $\Delta_{t} \approx \mathcal{O}(\mathrm{ns})$. Similarly, other exotic BSM physics could be the source a fixed time decay signature without relying on a heavy propagating sterile neutrino. The scenario described in refs. [44, 45] is one such case, it considers 

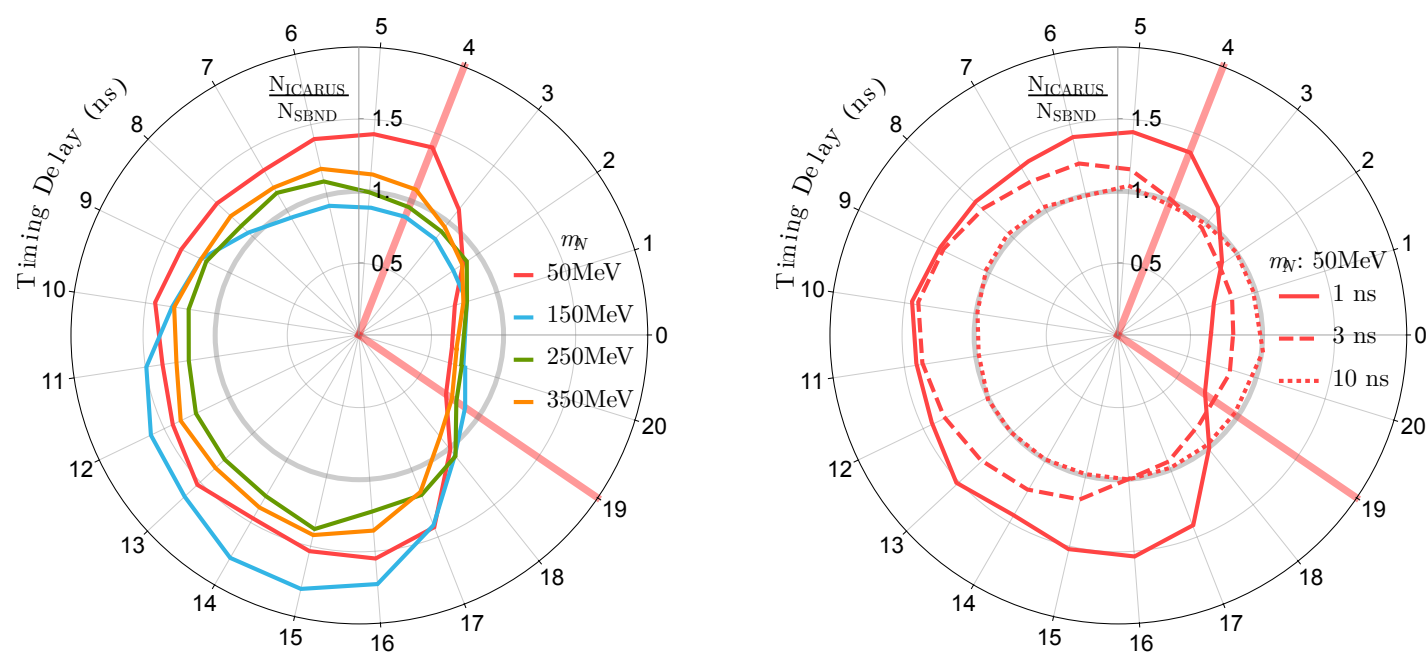

Figure 10. Left: ratio of arrival time spectra of $N \rightarrow \nu_{\alpha} e^{+} e^{-}$events in ICARUS to SBND after scaling out $1 / R^{2}$ flux dependence. If events were due to an unknown background with a fixed time delay after the neutrino beam, one would expect the ratio to be a constant value of 1 (shaded grey ring). As the sterile neutrinos have to travel approximately 6 times further to ICARUS than SBND, increasingly higher energy sterile neutrinos can leave the beam-bucket (red arc) and populate the inter-bucket region leading to the distinct signature. This assumes a timing resolution of $1 \mathrm{~ns}$. Right: the same as left for fixed $50 \mathrm{MeV}$ sterile neutrino with $1 \mathrm{~ns}$ (solid), $3 \mathrm{~ns}$ (dashed) and $10 \mathrm{~ns}$ (dotted) timing resolution showing the decreasing effect on the ratio.

a sterile neutrino produced inside the detector through neutral current scattering of an active neutrino. The heavy particle promptly decays, with a decay length of the order $\mathcal{O}(1) \mathrm{m}$, producing the visible signal. Although the sterile neutrinos are produced inside the detector with no timing delay from active-neutrino scattering, the finite lifetime of these particles could lead to events falling in the inter-bucket window.

However, in both the BSM scenarios as well as generic backgrounds with a fixed timing delay, the temporal spectra of event arrival time would be expected to be constant across all three detectors. The SBN program is perfectly designed to account for this, however, through its multiple detectors at different baselines, as if the excess is indeed due to heavy particle propagation, then the sterile neutrino would have to travel further to reach each subsequent detector. This leads to observable shifts in the arrival timing spectra at each experiment. In particular ICARUS, would be most suited to studying heavy particle propagation, as particles must travel approximately 6 times further before detection.

We show the consequences of this effect in figure 10 where the ratio of events at SBND and ICARUS are plotted as a function of time delay. A constant time delay would produce a ratio of unity, and curves that lie on the grey circle. We see a clear distortion in this ratio, with a generally low value inside the beam-bucket window and a larger value outside. Measuring this distortion would be definitive proof of the heavy particle having propagated the distance from target to detector and not merely being produced in situ. On the right panel of figure 10, we show how the attainable timing resolution affects this measurement. 
For a resolution of $10 \mathrm{~ns}$, there is no spectral difference, but distortion starts to be apparent for resolutions better than $1 \mathrm{~ns}$.

Assuming a positive signal is found and is identified as a heavy sterile neutrino decay thanks to the time delay, the temporal and energy analyses could be used to measure the heavy sterile neutrino mass. For an arrival time delay (behind a luminal or near luminal particle) over a distance $L$ denoted by $\Delta T$, the mass of a sterile neutrino with an energy $E$ can be reconstructed as

$$
m_{N}=E \sqrt{1-\frac{1}{\left(1+\frac{c \Delta T}{L}\right)^{2}}} .
$$

Exact knowledge of the deposited energy and time of flight would be sufficient to establish the mass, but of course these data are in most cases not available: energy and timing resolution impair the reconstruction, and many channels have missing energy from active neutrinos in the final state. Moreover, due to the cyclic nature of the BNB beam buckets, an observed event could have originated from any of the previous buckets in the current spill, and not just the one closest to the tagged event timing. As such the absolute time of flight is not known. Only the relative timing since the last bucket, $\Delta T$, is known and from this one can obtain up to 81 degenerate solutions for the sterile neutrino mass. Although absolute timing information could be found by studying the first few buckets for the onset of a signal, this would rely on precise absolute timing measurements between source and detector, and would also reduce the signal statistics by $\mathcal{O}(0.01)$ and we do not consider this information in the analysis. Given these limitations, we have studied how well $m_{N}$ could be reconstructed, using then energy and periodic time since last bucket $\Delta T$. We have generated Monte Carlo event data tagging each event by an arrival time, accounting for a systematic uncertainties on the time and energy measurement. We smear the true energy to represent detector effects as described above, and additionally smear the time of each event with a Gaussian of width $\sigma_{T} \approx 1 \mathrm{~ns}$ for SBND and ICARUS. We use the same Monte Carlo analysis and test statistic as in the temporal analysis above, expanded to include a binned energy spectra. The reconstructed mass is defined as the mass which minimises the test statistic $t_{m}$.

The results of our analysis are shown in figure 11. In both panels, we show the allowed region in reconstructed mass as a function of true sterile neutrino mass for an energy only analysis (dashed black lines), as well as for an energy and time-of-flight analysis (coloured bands). The left panel shows the results for the fully leptonic decay $N \rightarrow \nu e^{+} e^{-}$while the right panel shows our results for the semi-leptonic $N \rightarrow e^{ \pm} \pi^{\mp}$ channel. In the case of the 2-body $N \rightarrow e^{ \pm} \pi^{\mp}$ channel, resolution of approx $45 \mathrm{MeV}$ at $2 \sigma$ level is achievable for the entire range of sterile neutrino mass allowed. We estimate the $N \rightarrow \mu^{-} \pi^{+}$channel would be approximately $10 \%$ better due to the improved energy resolution possible when reconstructing muons in LAr. For these semi-leptonic decays the energy spectrum is very informative, as the parent particle's energy can be reconstructed from the invariant mass of the decay products' four-momenta. As such, we see temporal information only trivially improves the reconstruction of parent mass. In contrast, for the 3-body $N \rightarrow \nu e^{+} e^{-}$ channel, there is significant missing energy taken away by the active neutrino. In this case, 

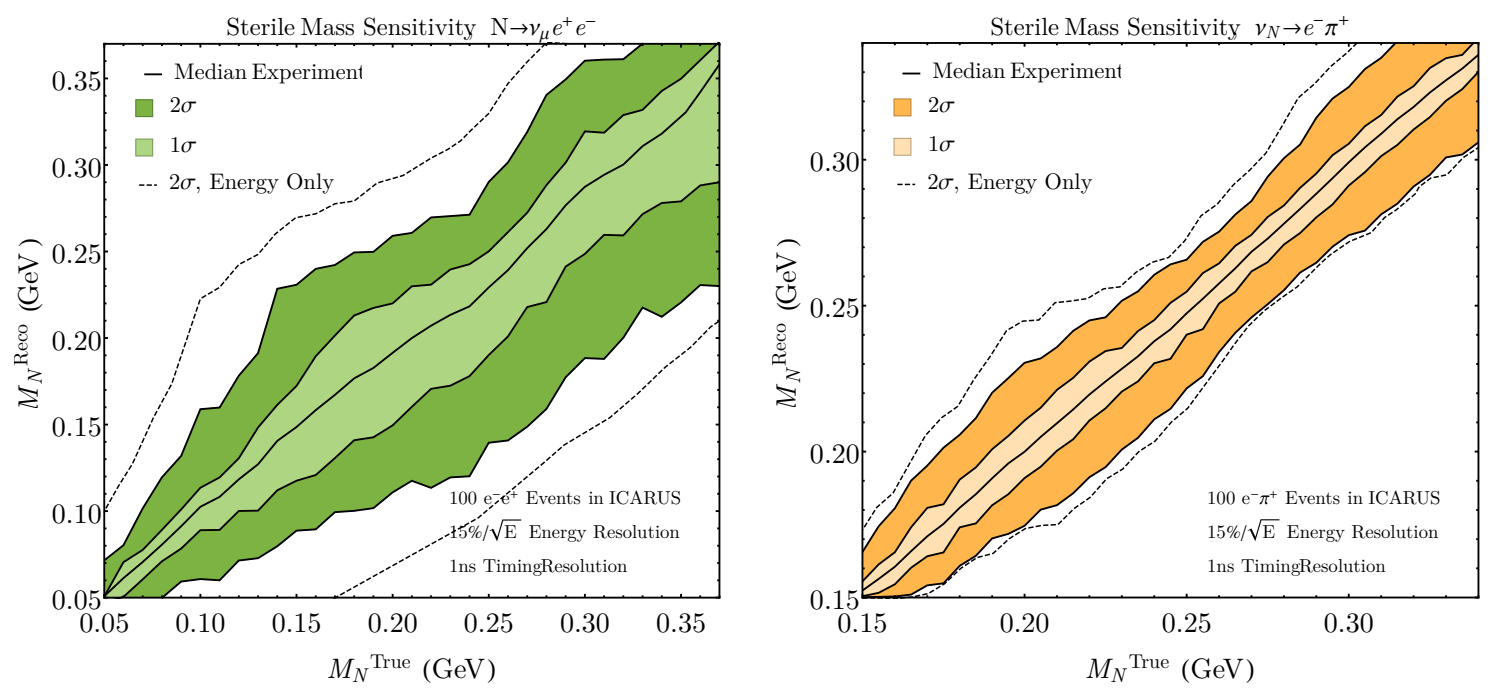

Figure 11. The reconstructed sterile neutrino mass as a function of true mass for energy only (dashed) and energy with timing information (solid). The left (right) panel assumes the decay $N \rightarrow \nu e^{+} e^{-}\left(N \rightarrow e^{ \pm} \pi^{\mp}\right)$. Both plots assume that 100 events are seen at ICARUS and that a $1 \mathrm{~ns}$ timing resolution is achieved.

timing information is much more valuable, almost halving the $2 \sigma$ mass range from around $300 \mathrm{MeV}$ to $150 \mathrm{MeV}$ for widest region of parameter space.

\section{Conclusions}

In this paper, we have studied the prospects for the measurement of $\mathrm{MeV}$-scale sterile neutrinos at the Fermilab Short-Baseline Neutrino program. MeV-scale neutral states would naturally be produced in the Booster Neutrino Beam from mixing-suppressed meson decays. To assess SBN's potential to constrain these models, we have estimated the dominant backgrounds and signals. Thanks to excellent particle identification and the distinctive kinematic properties of our signal, high levels of background suppression can be expected, allowing SBN to improve on the current bounds on decaying sterile neutrinos over most of the parameter space. We have shown that, in the absence of signal, SBN can place bounds on the active-sterile mixing-matrix elements of $\left|U_{e 4}\right|^{2} \leqslant 10^{-6}$ for $m_{N} \leqslant 33 \mathrm{MeV}$ and $\left|U_{\mu 4}\right|^{2} \leqslant 2 \times 10^{-8}$ for $m_{N} \leqslant 138 \mathrm{MeV}$ in the $N \rightarrow \nu e^{+} e^{-}$channels. For semi-leptonic decays, these bounds increase up to $\left|U_{e 4}\right|^{2} \leqslant 8 \times 10^{-10}$ for $m_{N} \leqslant 388 \mathrm{MeV}$ and up to $\left|U_{\mu 4}\right|^{2} \leqslant 7 \times 10^{-10}$ for $m_{N} \leqslant 493 \mathrm{MeV}$. The neutral pion decay channel, $N \rightarrow \nu \pi^{0}$, which may be the dominant decay mode for masses in the range $m_{\pi}^{0} \leqslant m_{N} \leqslant m_{\pi^{ \pm}}+m_{\mu}$, can be used to place bounds of around $\left|U_{\alpha 4}\right|^{2} \leqslant 3 \times 10^{-9}$. These can be expected to be the most stringent bounds placed by upcoming experiments in the region of parameter space studied in this paper. In particular, although significantly more sensitive for sterile neutrino masses above the kaon mass, SHiP produces weaker constraints than PS-191 for the case of a single sterile neutrino produced via kaon decay [30]. Any improvements in the bounds 
by SBN at lower sterile neutrino masses, $m_{N} \leqslant m_{K}$, will therefore complement the strong bounds placed by SHiP at higher masses. ${ }^{7}$

We have also discussed searches for non-minimal models of heavy sterile neutrino decay, which could lead to observable decays over a wide range of parameter space which is conventionally excluded if the branching ratios are assumed to arise from the minimal model. We have shown how to map existing minimal-model bounds onto non-minimal models and how bounds could be weakened in the case of specific enhancements to a decay channel. This motivates the search for particle decays in all channels over the full parameter space. We argue that some of these decay channels considered in this work are in fact poorly constrained by similar experiments, and show that SBN could place the first direct bounds on these processes.

We have commented in detail on the phenomenological role of timing information in this analysis. As well as providing a means of background suppression, nanosecond scale timing resolution could allow SBN to make direct measurements of the kinematic properties of heavy particle propagation. We have shown that if 100 events are seen at ICARUS, a 3.5 ns timing resolution would allow an observable timing delay to be established at $3 \sigma$ in $95 \%$ of experiments. We have seen that the unique design of SBN would allow for the distribution of event times to be compared between the nearest and farthest detectors, allowing for any model with a finite time delay to be excluded when compared to a propagating sterile neutrino model. We have also shown how timing information can be used in sterile neutrino mass reconstruction. For the decay $N \rightarrow \nu e^{+} e^{-}$, the inclusion of event timing information (with an assumed $1 \mathrm{~ns}$ resolution) can lead to the $2 \sigma$ allowed region being reduced from around $\pm 300 \mathrm{MeV}$ to $\pm 150 \mathrm{MeV}$.

We point out that this analysis is complementary to the central physics programme of SBN - studying eV-scale oscillating sterile neutrinos - and requires no additional detector or beam modifications. We have shown that SBN could contribute valuably to the search for sterile neutrino decays-in-flight, and moreover, if an anomalous signal is discovered, would play a central role in determining its origin, and the necessary extension of the SM.

\section{Acknowledgments}

We would like to thank Andrezj Szelc for his input at various stages of this work, and also to Jonathan Asaadi for helpful discussions and encouragement at the start of this project.

This work has been supported by the European Research Council under ERC Grant "NuMass" (FP7-IDEAS-ERC ENC-CG 617143), by the European Union FP7 ITNINVISIBLES (Marie Curie Actions, PITN-GA-2011-289442), as well as from the H2020 funded ELUSIVES ITN (H2020-MSCA-ITN-2015, GA- 2015-674896-ELUSIVES) and In-

\footnotetext{
${ }^{7} \mathrm{~A}$ purpose built SHiP-style experiment situated near the beam dump of a lower energy beam such as BNB could improve on this. Simple scaling arguments like those in table 1 predict event rates around $10^{4}$ times larger than PS-191, even for steriles $m_{N} \leqslant m_{K}$. This is not unexpected as SHiP consists of a huge decay volume designed to search for such particle decays. Such a simple scaling, however, does not take into consideration the complexity of working near an active beam dump.
} 
visiblePlus (H2020-MSCA-RISE-2015, GA-2015-690575-InvisiblesPlus). SP gratefully acknowledges partial support from the Wolfson Foundation and the Royal Society

\section{A Decay rates in the minimal model}

The decay rates in our study follow the notation of ref. [37]. We repeat them here in the interests of clarity. The dominant visible decay for sterile neutrinos with masses below the pion mass is into an electron positron pair. The total rate can be express as

$$
\begin{aligned}
\Gamma\left(N \rightarrow \nu_{\alpha} e^{+} e^{-}\right)= & \frac{G_{\mathrm{F}}^{2} m_{N}^{5}}{96 \pi^{3}}\left|U_{\alpha 4}\right|^{2}\left[\left(g_{L} g_{R}+\delta_{\alpha e} g_{R}\right) I_{1}\left(0, \frac{m_{e}}{m_{N}}, \frac{m_{e}}{m_{N}}\right)\right. \\
& \left.+\left(g_{L}^{2}+g_{R}^{2}+\delta_{\alpha e}\left(1+2 g_{L}\right)\right) I_{2}\left(0, \frac{m_{e}}{m_{N}}, \frac{m_{e}}{m_{N}}\right)\right],
\end{aligned}
$$

where $g_{L}=-1 / 2+\sin ^{2} \theta_{\mathrm{W}}, g_{R}=\sin ^{2} \theta_{\mathrm{W}}$ and

$$
\begin{aligned}
& I_{1}(x, y, z)=12 \int_{(x+y)^{2}}^{(1-z)^{2}} \frac{d s}{s}\left(s-x^{2}-y^{2}\right)\left(1+z^{2}-s\right) \sqrt{\lambda\left(s, x^{2}, y^{2}\right)} \sqrt{\lambda\left(1, s, z^{2}\right)}, \\
& I_{2}(x, y, z)=24 y z \int_{(y+z)^{2}}^{(1-x)^{2}} \frac{d s}{s}\left(1+x^{2}-s\right) \sqrt{\lambda\left(s, y^{2}, z^{2}\right)} \sqrt{\lambda\left(s, y^{2}, z^{2}\right)} \\
& \lambda(a, b, c)=a^{2}+b^{2}+c^{2}-2 a b-2 b c-2 c a .
\end{aligned}
$$

The decays into a charged lepton and a pion are given by

$$
\Gamma\left(N \rightarrow l^{ \pm} \pi^{\mp}\right)=\left|U_{l 4}\right|^{2} \frac{G_{\mathrm{F}}^{2} f_{\pi}^{2}\left|V_{u d}\right|^{2} m_{N}^{3}}{16 \pi} I\left(\frac{m_{l}^{2}}{m_{N}^{2}}, \frac{m_{\pi}^{2}}{m_{N}^{2}}\right),
$$

with

$$
I(x, y)=[(1+x+y)(1+x)-4 x] \lambda^{\frac{1}{2}}(1, x, y) .
$$

For $N \rightarrow e^{ \pm} \pi^{\mp}$ the kinematic function $I(x, y)$ produces only weak suppression $(I(x, y) \geqslant$ 0.5 ) for sterile neutrino masses above $m_{N} \gtrsim 150 \mathrm{MeV}$, whilst for $N \rightarrow \mu^{ \pm} \pi^{\mp}$ the equivalent threshold is $m_{N} \gtrsim 270 \mathrm{MeV}$.

\section{B Potential backgrounds}

In all channels a cut on vertex activity is applied as described in section 3.1 above. In this section, we provide a brief description of the backgrounds and additional cuts considered for each channel.

\section{B.1 $N \rightarrow e^{ \pm} \pi^{\mp}$}

The expected numbers of $e \pi$ events in the SBN detectors is significantly smaller than that of the $\mu \pi$ channel, as the fraction of intrinsic $\nu_{e}$ in the BNB beam is of $\mathcal{O}(1 \%)$ level in comparison to $\nu_{\mu}$. However, additional backgrounds to the $e \pi$ channel originate from the dominant $\nu_{\mu}$ component of the beam. CC $\nu_{\mu}$ events which contain an additional photon $(\mu+\gamma)$ have the potential to be be mis-identified as an $(\pi e)$ event, provided the muon has 
a sufficiently short track length, $<0.5 \mathrm{~m}$, in order to mimic a $\pi^{-}$. Additionally the photon must be mis-identified as an electron, with an efficiency of $94 \%$ using $d E / d x$ measurement, and must convert to an $e^{+} e^{-}$pair close enough to the interaction vertex as so there is no visible gap, $\leqslant 3 \mathrm{~cm}$. As energy resolution for EM showers is lower than muons, the invariant mass cut is less powerful requiring all events have an invariant mass below $500 \mathrm{MeV}$. A cut on the opening angle between lepton on meson, $\theta_{l \pi}<40^{\circ}$ as well as individual emission angles, $\theta_{l, \pi}<80^{\circ}$ further reduces the potential background. The $e^{-} \pi^{+}$channel is one of the cleanest channels under consideration in this paper, with 9,223 events in SBND reducing to 22 expected events post cuts, and with MicroBooNE and ICARUS expecting a reduction of $784(1,317)$ events to 2 and 3 respectively, with a signal efficiency of $71 \%$.

\section{B.2 $\quad N \rightarrow \nu_{\alpha} e^{+} e^{-}$and $N \rightarrow \gamma \nu_{\alpha}$}

A sufficiently boosted, and thus overlapping, $e^{+} e^{-}$pair is topologically indistinguishable from a converted photon in a LAr detector. Additional, non-topological measures such as the rate of energy loss, $d E / d x$, is also identical to a pair-converted photon. Thus we split this channel into two sub categories, when the $e^{+} e^{-}$is overlapping and photon-like, defined to be all events whose angular separation is $\leqslant 3^{\circ}[68]$ and all remaining separable two track events. The opening angle between the $e^{+} e^{-}$in a photon pair production scales roughly as $\approx m_{e} / E_{\gamma}$, with $3^{\circ}$ corresponding to $100 \mathrm{MeV}$ and used as a lower bound on energy. These backgrounds are also applicable to the $N \rightarrow \gamma \nu_{\alpha}$ channel.

The predominant source of backgrounds is the decay of a neutral pion in which a single photon is not resolved or escapes the fiducial volume. This background, however, is relatively isotropic in distribution in stark contrast to the very forward signal arising from the decay in flight of $N$. We place cuts on visible photon energy, $E_{\gamma} \geqslant 300 \mathrm{MeV}$, and angle of the observed photon to the beamline, $\theta_{\gamma} \leqslant 5^{\circ}$. This reduces the number of expected events from 42,580 (3,620 and 6,082) to 176 (46, and 110) events in SBND (MicroBooNE and ICARUS), while retaining a signal efficiency of $93 \%$.

For the opposite scenario both daughter electrons have a well defined and large separation and thus can cleanly be identified as two distinct single electron showers. There are few significant processes that produce high energy, distinguishable $e^{+} e^{-}$pairs. Instead the majority of the backgrounds are due to misidentifying two photons. We apply the same photon cuts as defined above. To further reject backgrounds in this channel, we apply a cut on the angle of separation between the distinct $e^{+} e^{-}$tracks of $\theta_{\text {sep }} \leqslant 40^{\circ}$ and total energy, $E_{e^{+}}+E_{e^{-}} \geqslant 100 \mathrm{MeV}$. This reduces the number of expected background events from 173 to 5 for SBND.

B.3 $N \rightarrow \pi^{0} \nu_{\alpha}$

Single neutral pions are produced in great numbers at the three SBN detectors, so the lack of any nuclear recoil is crucial in eliminating the incoherent neutral pion production background. Only events in which two photons convert inside the fiducial volume and reconstruct the pion invariant mass are accepted. We further require the reconstructed pion is within $10^{\circ}$ of the beamline and has an energy above $500 \mathrm{MeV}$. SBND expects $127,211 \pi^{0}$ events, of which $\approx 602$ survive all cuts with a signal efficiency of $32 \%$ for a 
sample $350 \mathrm{MeV}$ sterile neutrino. MicroBooNE (ICARUS) sees a similar reduction, from $10,813(18,172)$ events to 51 (86) post cuts.

\section{B.4 $N \rightarrow \nu \mu^{ \pm} \mu^{\mp}$}

The primary background for this channel is genuine $\nu_{\mu} \mathrm{CC}$ events in which a $\pi^{ \pm}$is also produced and is misidentified as a secondary muon. All pions with reconstructed tracks longer than $50 \mathrm{~cm}$ are considered a potential muon. After this track length cut, there is 2,044,380, 177,972 and 292,034 events in SBND, MicroBooNE and ICARUS respectively. As we cannot directly reconstruct the parent sterile neutrino mass or angle, we again rely on the kinematical difference between scattering events and decays. After these cuts, significant backgrounds remain, and we use a multivariate analysis, an adaptive boosted decision tree (BDT), in order to further reduce them. We use five parameters in this analysis, the energy and angle with respect to the beamline of each muon, as well as the angular separation between both muons. We take a minimum muon energy of $200 \mathrm{MeV}$. Cutting on the BDT response variable allows for background efficiency of $0.13 \%$, with a corresponding signal efficiency of $44 \%$. This allows for a $S / \sqrt{S+B} \approx 8$ with approximately 1000 sterile neutrino events. Similar performance is achievable at MicroBooNE and ICARUS, with 117,972 and 292,034 events being reduced to 534 and 876 events respectively.

\section{B.5 $N \rightarrow \nu e^{ \pm} \mu^{\mp}$}

We consider here two potential sources of backgrounds: the first derives from true $\nu_{\mu} \mathrm{CC}$ events in which a single photon, either from nuclear processes or from the decay of a $\pi^{0}$ in which only photon converts inside the fiducial volume, subsequently mimics the electron. We apply the same cuts on the photon as in previous channels. Secondly we consider intrinsic $\nu_{e}$ CC events in which a final state $\pi^{ \pm}$is misidentified as a muon due to a long ( $\geqslant 50$ $\mathrm{cm})$ track in the TPC. In conjunction with the requirement of no visible scattering vertex we expect 7,103, 618 and 1,014 events in SBND, MicroBooNE and ICARUS, respectively. To reduce this further we employ the same multivariate analysis as described for the $N \rightarrow$ $\nu \mu \mu$ channel above, assuming a representative $250 \mathrm{MeV}$ sterile neutrino decaying. A cut on the BDT allows for a background efficiency of $0.5 \%$, signal efficiency of $36 \%$ with a resultant $S / \sqrt{S+B}$ of 7.9. For MicroBooNE and ICARUS the backgrounds, 618 and 1,014 respectively, can be brought down to sub 10 events.

\section{B.6 Non-Beam related backgrounds}

Cosmogenic events are a potential source of background for any analysis at SBN. In the case of cosmic muons, Icarus expects to see approximately $2.5 \times 10^{6}$ cosmic events in the 211 second beam spill, which are reduced to approximately 5 events expected after utilising the spill structure, scintillation light patterns and cuts on $\frac{d E}{d x}[14]$. Alongside this impressive cosmic rejection, our signal events are focused heavily along the beamline, hence we do not expect cosmics to be a major source of background to any channel. In situ beam-off cosmic studies will also allow potential backgrounds to be extremely well understood by the time of an analysis such as this, and for these reasons, we do not include cosmogenic backgrounds in our analysis. 


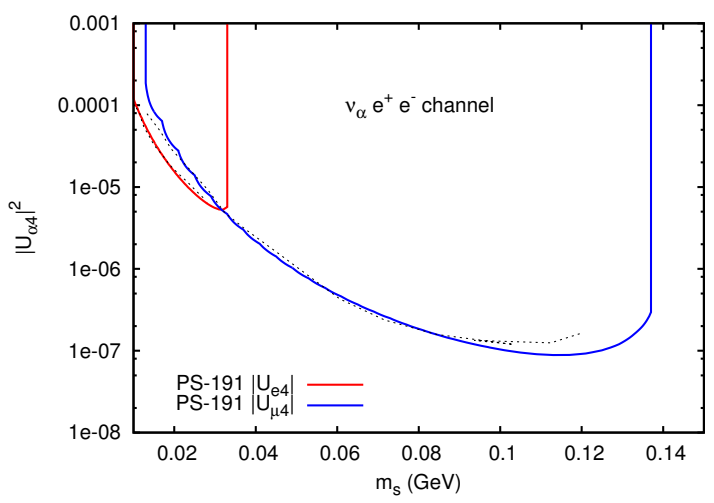

Figure 12. Estimated bounds on $\left|U_{e 4}\right|^{2}$ and $\left|U_{\mu 4}\right|^{2}$ for a Dirac heavy sterile neutrino decaying to $\nu_{\alpha} e^{+} e^{-}$at PS-191. The dotted black lines are the 90\% CL results as published by PS-191, and the blue and red curves are the results of our simulation for $0.86 \times 10^{19}$ POT.

\section{PS-191 bound reproduction}

As a consistency check of our methodology we reproduce here the bounds on $\left|U_{e 4}\right|$ and $\left|U_{\mu 4}\right|$ for sterile masses below $m_{\pi}$ as published by PS-191. The detector geometry is assumed to be $6 \mathrm{~m} \times 3 \mathrm{~m} \times 12 \mathrm{~m}$ and was located $128 \mathrm{~m}$ downstream of the Beryllium target using 19.2 GeV protons from the PS proton beam. Fluxes of all neutrinos produced from pion sources at PS-191 were obtained from [69]. No accurate kaon sources could be obtained and as such only low mass bounds are reproduced here. It must be noted that PS-191 ignored all neutral current contributions to $N \rightarrow \nu_{\alpha} e^{+} e^{-}$and assumed the sterile neutrinos were Dirac particles; the effect of this is that the bounds published are not directly comparable to the minimal model discussed above, and must be scaled appropriately. The bounds reproduced are in good agreement with published data, as we show in figure 12 .

Open Access. This article is distributed under the terms of the Creative Commons Attribution License (CC-BY 4.0), which permits any use, distribution and reproduction in any medium, provided the original author(s) and source are credited.

\section{References}

[1] Super-Kamiokande collaboration, Y. Fukuda et al., Evidence for oscillation of atmospheric neutrinos, Phys. Rev. Lett. 81 (1998) 1562 [hep-ex/9807003] [INSPIRE].

[2] P. Minkowski, $\mu \rightarrow$ er at a Rate of One Out of $10^{9}$ Muon Decays?, Phys. Lett. B 67 (1977) 421 [INSPIRE].

[3] M. Gell-Mann, P. Ramond and R. Slansky, Complex Spinors and Unified Theories, Conf. Proc. C 790927 (1979) 315 [arXiv: 1306.4669] [INSPIRE].

[4] R.N. Mohapatra and G. Senjanović, Neutrino Mass and Spontaneous Parity Violation, Phys. Rev. Lett. 44 (1980) 912 [INSPIRE].

[5] A. Zee, A Theory of Lepton Number Violation, Neutrino Majorana Mass and Oscillation, Phys. Lett. B 93 (1980) 389 [Erratum ibid. B 95 (1980) 461] [INSPIRE]. 
[6] K.S. Babu, Model of 'Calculable' Majorana Neutrino Masses, Phys. Lett. B 203 (1988) 132 [INSPIRE].

[7] N. Arkani-Hamed, S. Dimopoulos, G.R. Dvali and J. March-Russell, Neutrino masses from large extra dimensions, Phys. Rev. D 65 (2001) 024032 [hep-ph/9811448] [INSPIRE].

[8] S. Gariazzo, C. Giunti, M. Laveder, Y.F. Li and E.M. Zavanin, Light sterile neutrinos, J. Phys. G 43 (2016) 033001 [arXiv: 1507.08204] [InSPIRE].

[9] LSND collaboration, A. Aguilar-Arevalo et al., Evidence for neutrino oscillations from the observation of anti-neutrino(electron) appearance in a anti-neutrino(muon) beam, Phys. Rev. D 64 (2001) 112007 [hep-ex/0104049] [INSPIRE].

[10] MiniBoonE collaboration, A.A. Aguilar-Arevalo et al., A Combined $\nu_{\mu} \rightarrow \nu_{e}$ and $\bar{\nu}_{\mu} \rightarrow \bar{\nu}_{e}$ Oscillation Analysis of the MiniBooNE Excesses, Phys. Rev. Lett. 110 (2013) 161801 [arXiv: 1207.4809] [INSPIRE].

[11] MiniBoonE collaboration, A.A. Aguilar-Arevalo et al., Unexplained Excess of Electron-Like Events From a 1-GeV Neutrino Beam, Phys. Rev. Lett. 102 (2009) 101802 [arXiv: 0812.2243] [INSPIRE].

[12] J. Kopp, P.A.N. Machado, M. Maltoni and T. Schwetz, Sterile Neutrino Oscillations: The Global Picture, JHEP 05 (2013) 050 [arXiv: 1303.3011] [INSPIRE].

[13] J.M. Conrad, C.M. Ignarra, G. Karagiorgi, M.H. Shaevitz and J. Spitz, Sterile Neutrino Fits to Short Baseline Neutrino Oscillation Measurements, Adv. High Energy Phys. 2013 (2013) 163897 [arXiv: 1207.4765] [INSPIRE].

[14] LAr1-ND, ICARUS-WA104, MicroBooNE collaborations, M. Antonello et al., A Proposal for a Three Detector Short-Baseline Neutrino Oscillation Program in the Fermilab Booster Neutrino Beam, arXiv:1503.01520 [INSPIRE].

[15] C. Rubbia, The liquid argon time projection chamber: a new concept for neutrino detector, (1977) [INSPIRE].

[16] E.K. Akhmedov and A. Yu. Smirnov, Paradoxes of neutrino oscillations, Phys. Atom. Nucl. 72 (2009) 1363 [arXiv:0905.1903] [INSPIRE].

[17] E.D. Church, LArSoft: A Software Package for Liquid Argon Time Projection Drift Chambers, arXiv:1311.6774 [INSPIRE].

[18] J.S. Marshall and M.A. Thomson, The Pandora Software Development Kit for Pattern Recognition, Eur. Phys. J. C 75 (2015) 439 [arXiv:1506.05348] [INSPIRE].

[19] M. Sorel, Expected performance of an ideal liquid argon neutrino detector with enhanced sensitivity to scintillation light, 2014 JINST 9 P10002 [arXiv:1405.0848] [INSPIRE].

[20] M. Antonello et al., Precise 3D track reconstruction algorithm for the ICARUS T600 liquid argon time projection chamber detector, Adv. High Energy Phys. 2013 (2013) 260820 [arXiv: 1210.5089] [INSPIRE].

[21] Argoneut, MicroBoone collaborations, A. Szelc, Recent Results from ArgoNeuT and Status of MicroBooNE, talk given at Neutrino, 2014 (2014).

[22] G. Bernardi et al., Search for Neutrino Decay, Phys. Lett. B 166 (1986) 479 [InSPIRE].

[23] G. Bernardi et al., Further limits on heavy neutrino couplings, Phys. Lett. B 203 (1988) 332 [INSPIRE]. 
[24] R. Adhikari et al., A White Paper on keV Sterile Neutrino Dark Matter, JCAP 01 (2017) 025 [arXiv: 1602.04816] [INSPIRE].

[25] T. Asaka and M. Shaposhnikov, The nuMSM, dark matter and baryon asymmetry of the universe, Phys. Lett. B 620 (2005) 17 [hep-ph/0505013] [INSPIRE].

[26] WA66 collaboration, A.M. Cooper-Sarkar et al., Search for Heavy Neutrino Decays in the BEBC Beam Dump Experiment, Phys. Lett. B 160 (1985) 207 [inSPIRE].

[27] CHARM collaboration, F. Bergsma et al., A Search for Decays of Heavy Neutrinos in the Mass Range 0.5 GeV to 2.8 GeV, Phys. Lett. B 166 (1986) 473 [InSPIRE].

[28] NuTeV, E815 collaborations, A. Vaitaitis et al., Search for neutral heavy leptons in a high-energy neutrino beam, Phys. Rev. Lett. 83 (1999) 4943 [hep-ex/9908011] [INSPIRE].

[29] SHiP collaboration, M. Anelli et al., A facility to Search for Hidden Particles (SHiP) at the CERN SPS, arXiv:1504.04956 [INSPIRE].

[30] S. Alekhin et al., A facility to Search for Hidden Particles at the CERN SPS: the SHiP physics case, Rept. Prog. Phys. 79 (2016) 124201 [arXiv: 1504.04855] [InSPIRE].

[31] D. Gorbunov and M. Shaposhnikov, How to find neutral leptons of the $\nu$ MSM?, JHEP 10 (2007) 015 [Erratum ibid. 11 (2013) 101] [arXiv:0705.1729] [INSPIRE].

[32] T. Asaka, S. Eijima and A. Watanabe, Heavy neutrino search in accelerator-based experiments, JHEP 03 (2013) 125 [arXiv:1212.1062] [INSPIRE].

[33] LBNE collaboration, C. Adams et al., The Long-Baseline Neutrino Experiment: Exploring Fundamental Symmetries of the Universe, arXiv:1307.7335 [INSPIRE].

[34] D. Hernandez and A. Yu. Smirnov, Active to sterile neutrino oscillations: Coherence and MINOS results, Phys. Lett. B 706 (2012) 360 [arXiv:1105.5946] [INSPIRE].

[35] MiniBoonE collaboration, A.A. Aguilar-Arevalo et al., The Neutrino Flux prediction at MiniBooNE, Phys. Rev. D 79 (2009) 072002 [arXiv:0806.1449] [InSPIRE].

[36] R.E. Shrock, General Theory of Weak Leptonic and Semileptonic Decays. 1. Leptonic Pseudoscalar Meson Decays, with Associated Tests For and Bounds on, Neutrino Masses and Lepton Mixing, Phys. Rev. D 24 (1981) 1232 [INSPIRE].

[37] A. Atre, T. Han, S. Pascoli and B. Zhang, The Search for Heavy Majorana Neutrinos, JHEP 05 (2009) 030 [arXiv: 0901.3589] [INSPIRE].

[38] R.E. Shrock, General Theory of Weak Processes Involving Neutrinos. 2. Pure Leptonic Decays, Phys. Rev. D 24 (1981) 1275 [inSPIRE].

[39] P.B. Pal and L. Wolfenstein, Radiative Decays of Massive Neutrinos, Phys. Rev. D 25 (1982) 766 [INSPIRE].

[40] F. del Aguila, S. Bar-Shalom, A. Soni and J. Wudka, Heavy Majorana Neutrinos in the Effective Lagrangian Description: Application to Hadron Colliders, Phys. Lett. B 670 (2009) 399 [arXiv:0806.0876] [INSPIRE].

[41] A. Aparici, K. Kim, A. Santamaria and J. Wudka, Right-handed neutrino magnetic moments, Phys. Rev. D 80 (2009) 013010 [arXiv:0904.3244] [InSPIRE].

[42] Virgo, Ligo Scientific collaborations, S. Bhattacharya and J. Wudka, Dimension-seven operators in the standard model with right handed neutrinos, Phys. Rev. D 94 (2016) 055022 [arXiv: 1505. 05264] [INSPIRE]. 
[43] S. Weinberg, Baryon and Lepton Nonconserving Processes, Phys. Rev. Lett. 43 (1979) 1566 [INSPIRE].

[44] S.N. Gninenko, The MiniBooNE anomaly and heavy neutrino decay, Phys. Rev. Lett. 103 (2009) 241802 [arXiv:0902.3802] [INSPIRE].

[45] S.N. Gninenko, A resolution of puzzles from the LSND, KARMEN and MiniBooNE experiments, Phys. Rev. D 83 (2011) 015015 [arXiv:1009.5536] [InSPIRE].

[46] L. Duarte, I. Romero, J. Peressutti and O.A. Sampayo, Effective Majorana neutrino decay, Eur. Phys. J. C 76 (2016) 453 [arXiv:1603.08052] [InSPIRE].

[47] B. Batell, M. Pospelov and B. Shuve, Shedding Light on Neutrino Masses with Dark Forces, JHEP 08 (2016) 052 [arXiv: 1604.06099] [inSPIRE].

[48] R. Foot, X.G. He, H. Lew and R.R. Volkas, Model for a light Z-prime boson, Phys. Rev. D 50 (1994) 4571 [hep-ph/9401250] [INSPIRE].

[49] D.I. Britton et al., Improved search for massive neutrinos in $\pi^{+} \rightarrow e^{+} \nu$ decay, Phys. Rev. D 46 (1992) R885.

[50] D.I. Britton et al., Measurement of the $\pi^{+} \rightarrow e^{+} \nu$ branching ratio, Phys. Rev. Lett. 68 (1992) 3000 [INSPIRE].

[51] P. Coloma, B.A. Dobrescu, C. Frugiuele and R. Harnik, Dark matter beams at LBNF, JHEP 04 (2016) 047 [arXiv: 1512.03852] [INSPIRE].

[52] MiniBooNE collaboration, R. Dharmapalan et al., Low Mass WIMP Searches with a Neutrino Experiment: A Proposal for Further MiniBooNE Running, arXiv:1211.2258 [INSPIRE].

[53] NA3 collaboration, J. Badier et al., Mass and Lifetime Limits on New Longlived Particles in 300-GeV/cr- Interactions, Z. Phys. C 31 (1986) 21 [INSPIRE].

[54] B. Fields and S. Sarkar, Big-Bang nucleosynthesis (2006 Particle Data Group mini-review), astro-ph/0601514 [INSPIRE].

[55] A.D. Dolgov, S.H. Hansen, G. Raffelt and D.V. Semikoz, Heavy sterile neutrinos: Bounds from big bang nucleosynthesis and SN1987A, Nucl. Phys. B 590 (2000) 562 [hep-ph/0008138] [INSPIRE].

[56] A.D. Dolgov, S.H. Hansen, G. Raffelt and D.V. Semikoz, Cosmological and astrophysical bounds on a heavy sterile neutrino and the KARMEN anomaly, Nucl. Phys. B 580 (2000) 331 [hep-ph/0002223] [INSPIRE].

[57] C. Andreopoulos et al., The GENIE Neutrino Monte Carlo Generator, Nucl. Instrum. Meth. A 614 (2010) 87 [arXiv:0905.2517] [InSPIRE].

[58] Argoneut collaboration, R. Acciarri et al., Detection of back-to-back proton pairs in charged-current neutrino interactions with the ArgoNeuT detector in the NuMI low energy beam line, Phys. Rev. D 90 (2014) 012008 [arXiv:1405.4261] [INSPIRE].

[59] MiniBoonE collaboration, M.O. Wascko, Charged current single pion cross section measurement at MiniBooNE, in proceedings of the 4 th International Workshop on Neutrino-Nucleus Interactions in the Few-GeV Region, Okayama, Japan, 26-29 September 2005 [Nucl. Phys. Proc. Suppl. 159 (2006) 50] [hep-ex/0602050] [InSPIRE].

[60] MINERvA collaboration, A. Higuera et al., Measurement of Coherent Production of $\pi^{ \pm}$in Neutrino and Antineutrino Beams on Carbon from $E_{\nu}$ of 1.5 to $20 \mathrm{GeV}$, Phys. Rev. Lett. 113 (2014) 261802 [arXiv:1409.3835] [inSPIRE]. 
[61] D. Rein and L.M. Sehgal, PCAC and the Deficit of Forward Muons in pit Production by Neutrinos, Phys. Lett. B 657 (2007) 207 [hep-ph/0606185] [InSPIRE].

[62] R. Acciarri et al., Summary of the Second Workshop on Liquid Argon Time Projection Chamber Research and Development in the United States, 2015 JINST 10 T07006 [arXiv: 1504.05608] [INSPIRE].

[63] MicroBoone Collaboration, M. Toups, First Results From MicroBooNE, Neutrino London, July, 2016.

[64] ISTRA+ collaboration, V.A. Duk et al., Search for Heavy Neutrino in $K^{--}>\mu^{-} \nu_{h}\left(\nu_{h}->\nu \gamma\right)$ decay at ISTRA + Setup, Phys. Lett. B 710 (2012) 307 [arXiv:1110.1610] [INSPIRE].

[65] S.N. Gninenko and N.V. Krasnikov, Limits on the magnetic moment of sterile neutrino and two photon neutrino decay, Phys. Lett. B 450 (1999) 165 [hep-ph/9808370] [INSPIRE].

[66] G.J. Feldman and R.D. Cousins, A Unified approach to the classical statistical analysis of small signals, Phys. Rev. D 57 (1998) 3873 [physics/9711021] [InSPIRE].

[67] Particle Data Group collaboration, K.A. Olive et al., Review of Particle Physics, Chin. Phys. C 38 (2014) 090001 [inSPIRE].

[68] J.B. Spitz, Measuring Muon-Neutrino Charged-Current Differential Cross sections with a Liquid Argon Time Projection Chamber, Ph.D. Thesis, Yale University (2011).

[69] G. Bernardi, Mesure du flux de $\nu_{e}$ dans le faisceau de neutrinos de basse energie du PS: intrepretation dans le cadre d'une oscillation $\nu_{\mu} \rightarrow \nu_{e}$, Ph.D. Thesis, Unversité Pierre et Marie Curie (1985). 